\title{
Applied Mechatronics: Designing a Sliding Mode Controller for Active Suspension System
}

\author{
Aydin Azizi $\mathbb{D}^{1}$ and Hamed Mobki \\ ${ }^{1}$ School of Engineering, Computing and Mathematics, Oxford Brookes University, Wheatley Campus, Oxford OX33 1HX, UK \\ ${ }^{2}$ Department of Engineering, German University of Technology in Oman, Muscat, Oman \\ Correspondence should be addressed to Aydin Azizi; aydin.azizi@brookes.ac.uk
}

Received 19 November 2020; Revised 24 March 2021; Accepted 24 April 2021; Published 7 May 2021

Academic Editor: Matilde Santos

Copyright (C 2021 Aydin Azizi and Hamed Mobki. This is an open access article distributed under the Creative Commons Attribution License, which permits unrestricted use, distribution, and reproduction in any medium, provided the original work is properly cited.

\begin{abstract}
The suspension system is referred to as the set of springs, shock absorbers, and linkages that connect the car to the wheel system. The main purpose of the suspension system is to provide comfort for the passengers, which is created by reducing the effects of road bumpiness. It is worth noting that reducing the effects of such vibrations also diminishes the noise and undesirable sound as well as the effects of fatigue on mechanical parts of the vehicle. Due to the importance of the abovementioned issues, the objective of this article is to reduce such vibrations on the car by implementing an active control method on the suspension system. For this purpose, a conventional first-order sliding mode controller has been designed for stochastic control of the quarter-car model. It is noteworthy that this controller has a significant ability to overcome the stochastic effects, uncertainty, and deal with nonlinear factors. To design a controller, the governing dynamical equation of the quarter-car system has been presented by considering the nonlinear terms in the springs and shock absorber, as well as taking into account the uncertainty factors in the system and the actuator. The design process of the sliding mode controller has been presented and its stability has been investigated in terms of the Lyapunov stability. In the current research, road surface variations are considered as Gaussian white noise. The dynamical system behavior for controlled and uncontrolled situations has been simulated and the extracted results have been presented. Besides, the effects of existing uncertainty in the suspension system and actuator have been evaluated and controller robustness has been checked. Also, the obtained quantitative and qualitative compressions have been presented. Moreover, the effect of controller parameters on the basin of attraction set and its extensiveness has been assessed. The achieved results have indicated the good performance and significant robustness of the designed controller to stabilize the suspension system and mitigate the effects of road bumpiness in the presence of uncertainty and noise factors.
\end{abstract}

\section{Introduction}

In recent decades, the automotive industry has witnessed rapid progress, and several studies in the cases of design of car shape based on aerodynamic optimization [1], [2], optimization of the air intake system of the engine [3], investigation of sound quality for passenger car [4], stress analysis and design improvement of door hinge for compact cars [5], study of fuel consumption for various driving styles in conventional and hybrid electric vehicles [6, 7], design optimization of the cowl cross bar-light [8], investigation of a rear independent suspension for light vehicle [9], and study of nonlinear control of suspension system [10-12] have been conducted to develop and optimize different aspects of vehicle performance. The suspension system is one of the main components of a car which plays an important role in providing passenger comfort. The suspension system is generally divided into three classes of passive, semiactive, and active. The main task of this system is to reduce the amplitude and unwanted effects of vibrations imposed by road surface roughness on the car's chassis. In addition to providing comfort, reducing such impacts plays an important role in the transportation of goods and military industries. For example, when transporting fruits, animals, or delicate materials, vibrations with large amplitudes are a matter of concern. Reducing vibrations is also essential for 
accurate aiming of military vehicles. Furthermore, damped vibrations improve vehicle steering [13], braking performance, and energy consumption. Additionally, fatigue can be prevented in many parts of the vehicle such as the body and engine.

As the most effective method for reducing the impact of chassis vibrations, active suspension has been extensively studied in the literature. Rizvi et al. [14] have utilized robust control techniques for active control of a car suspension system. Wang et al. [15] presented a new model-free fractional-order sliding mode control based on an Extended State Observer (ESO) for quarter-car active suspension systems.

Mardani [16] has investigated energy harvesting, handling, and ride comfort tradeoff between passive and active suspension systems, using a PID controller. Khodadadi and Ghadiri [17] have utilized PID, fuzzy logic, and Hinf controllers to analyze the suspension system. Kumar et al. [18] applied a fractional-order fuzzy PD controller to analyze the car suspension system. In [19], the adaptive neurofuzzy inference system control has been utilized to analyze the active suspension system. Other control strategies related to the control and optimization of industrial systems that can be also applied to the control of the suspension system have been presented in [20], [21].

Nonlinear factors and parameters of spring and the shock absorber are the main points to be taken into consideration. Furthermore, unmodeled uncertainties in the suspension and other mechanical systems can make it difficult to control the system. In addition, the actuator can also include uncertainty or defects, where defects are inherently considered a type of uncertainty. The abovementioned issues, that is, nonlinearity, uncertainty, and disturbances, complicate the design procedure of the active suspension or controller.

To reduce the effects of uncertainty and disturbances, robust control methods are needed since not all control methods are capable of providing stabilization [22-24]. Sliding mode controller and Hinf are two of the most important robust control methods offering a favorable performance in reducing the effects of noise and uncertainty [25-27]. It is necessary to determine the norms related to uncertainties and disturbances, in the Hinf method. But for the SMC method, it is only necessary to have the upper and lower bands of these terms. Also, in the Hinf method, there is no problem called chatting phenomenon but in the SMC method, there is such a phenomenon [28, 29]. However, with procedures, the effects of this phenomenon can be reduced. In general, considering that, in this study, only the upper and lower bands of uncertainties and disturbances are available, the sliding mode method has been utilized to control. It is noteworthy that, in the recent study [30], the SMC method was used to design the controller and the Hinf method was used to determine the optimal feedback gain. This study shows that the SMC and Hinf methods are combined and used simultaneously to control the dynamical systems [31].

Given the importance of controlling and modeling the car suspension system, this study discusses the control of this system and the evaluation of its stability by means of a basin attraction set. As mentioned before, in addition to the linear term, springs and shock absorbers include nonlinear terms. Furthermore, these parts as well as actuators can also include uncertainties. Therefore, for the precise simulation of the system, all of the abovementioned parameters are considered in this work. Due to the high capability of the sliding mode method in controlling the nonlinear systems and overcoming the effects of uncertainty and disturbance, this method is considered to control it. In addition to the discussion of structure control, the discussion of the basin of attraction set is also examined at the end of the results section. Moreover, the effects of uncertainty in the system and actuator together with the effects of controller parameters on the dynamical behavior of the system and the region of attraction set are investigated. In this paper, based on the authors' previous related research [31-34], road surface variations are considered as Gaussian white noise. Obviously, the use of fuzzy-sliding mode [35], neural-sliding mode, or second-order sliding mode controller along with more complexity and calculations can increase the efficiency of the control system or reduce the effects of chattering. In addition to these cases, it is even possible to increase the scope of research by considering the existence of faults in the structure or investigating the fault-tolerant problem in the presence of time-delayed Markov jump [36] and examine the control of the faulty system. However, considering that the main purpose of this paper is reducing uncertainty effects and considering nonlinear factors in dynamic modeling and evaluating stability, so only the common first-order sliding mode scheme is recruited to analyze of results.

\section{Model Description}

Before presenting the controller design and mathematical molding of the quarter-car suspension system, the geometric and physical model of this system is presented here.

Considerable research has been conducted by assuming the suspension system in the linear range [37]. However, in a more precise point of view, considering the actual situation of spring and damper between car and the tire [38], as well as considering that springs and dampers include nonlinear terms, it is more accurate to consider these nonlinear terms in simulation procedure. Furthermore, tire stiffness consists of a set of linear and nonlinear terms. Therefore, in this paper, to increase the accuracy of the physical model, nonlinear terms of springs and damper also participate in physical modeling.

Figure 1 shows the nonlinear quarter-car model used in this study. As shown, the system includes two masses of $M_{1}$ and $M_{2}$. Sprung mass $M_{1}$ represents a quarter of the equivalent mass of the vehicle and passengers, while the unsprung mass $M_{2}$ represents the mass of the wheel. As shown in the figure, a spring, a shock absorber, and an actuator are present between masses $M_{1}$ and $M_{2}$.

Depending on how the spring and shock absorber are linked between the tire and car, these parts may be considered with nonlinear terms. One such model is the Machperson model [38]. In addition, as mentioned earlier, 


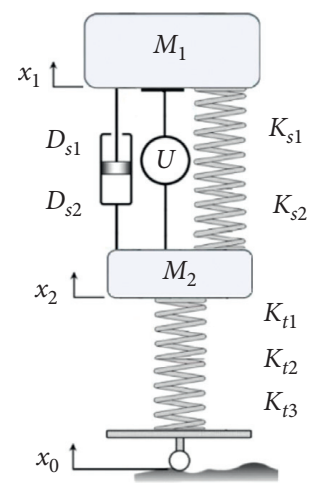

FIGURE 1: Schematic view of active quarter-car suspension model.

springs and shock absorbers naturally include nonlinear terms; although these nonlinear terms may have a diminutive effect, considering them increases the simulation accuracy.
The spring and shock absorbers consist of linear and nonlinear where $K_{s 1}$ and $D_{s 1}$ represent the constants for the linear terms of the spring and shock absorber, respectively. Moreover, $K_{s 2}$ and $D_{s 2}$ express the nonlinear spring and shock absorber coefficients. The actuator provides the force needed for controlling the system. The tire exhibits a stiffness and, therefore, is modeled as a spring connected to mass $M_{2}$ from the top, while its other end is in contact with the road surface from the bottom. The coefficient $K_{t 1}$ represents the constant for the linear term of the tire stiffness, while the coefficients $K_{t 2}$ and $K_{t 3}$ denote the constants for its nonlinear terms. The vertical displacement of masses $M_{1}$ and $M_{2}$ is also shown and indicated by $x_{1}$ and $x_{2}$, while $x_{0}$ represents the vertical variations in the pavement.

\section{Mathematical Modeling}

3.1. Governing Dynamic Equation of Quarter-Car System. The governing dynamic equation of the system shown in Figure 1 can be represented as follows:

$$
\begin{aligned}
& M_{1} \ddot{x}_{1}+K_{s 1}\left(x_{1}-x_{2}\right)+K_{s 2}\left(x_{1}-x_{2}\right)^{3}+D_{s 1}\left(\dot{x}_{1}-\dot{x}_{2}\right)+D_{s 2}\left(\dot{x}_{1}-\dot{x}_{2}\right)^{2}+f_{1}(x, t)=h(x, t) u \\
& M_{2} \ddot{x}_{2}-K_{s 1}\left(x_{1}-x_{2}\right)-K_{s 2}\left(x_{1}-x_{2}\right)^{3}-D_{s 1}\left(\dot{x}_{1}-\dot{x}_{2}\right)-D_{s 2}\left(\dot{x}_{1}-\dot{x}_{2}\right)^{2} \\
& +K_{t 1}\left(x_{2}-x_{0}\right)+K_{t 2}\left(x_{2}-x_{0}\right)^{2}-K_{t 3}\left(x_{2}-x_{0}\right)^{3}+f_{2}(x, t)=-h(x, t) u
\end{aligned}
$$

where $\dot{x}_{1}$ and $\dot{x}_{2}$ represent the time derivatives of $x_{1}$ and $x_{2}$ and $u$ is the actuator force, in which its magnitude is specified by the controller. Given that actuators are not usually considered to be ideal and include uncertainty, the bounded function $h(x, t)$ was considered to model the actuator uncertainty. Note that the minimum value of $h(x, t)$ is positive and denoted by $h_{0}$. Functions $f_{1}(x, t)$ and $f_{2}(x, t)$ also signify the unmodeled uncertainty of the system that has a known upper limited value.

3.2. Sliding Mode Controller Design. In this section, the sliding mode controller has been designed to stabilize the vertical movement of mass $M_{1}$. The goal is to stabilize the vertical movement of $M_{1}$, that is, $x_{1}$, and to track $x_{d}$. To this end, the tracking error is considered as $e=x_{1}-x_{d}$ [39]. The tracking error is also expressed by $\dot{e}=\dot{x}_{1}-\dot{x}_{d}$. Assuming $e=e_{1}$, the dynamic of tracking error can be expressed as

$$
\begin{aligned}
& \left\{\begin{array}{l}
e=x_{1}-x_{d}, \\
\dot{e}=\dot{x}_{1}-\dot{x}_{d},
\end{array}\right. \\
& \text { Or } \\
& \left\{\begin{array}{l}
\dot{e}_{1}=e_{2}, \\
\dot{e}_{2}=\ddot{x}_{1}
\end{array}-\ddot{x}_{d} .\right.
\end{aligned}
$$

By determining $\ddot{x}_{1}$ from equation (1) and substituting it in equation (2), the error dynamics can be written as

$$
\left\{\begin{array}{l}
\dot{e}_{1}=e_{2}, \\
\dot{e}_{2}=\frac{1}{M_{1}}\left[-K_{s 1}\left(x_{1}-x_{2}\right)-K_{s 2}\left(x_{1}-x_{2}\right)^{3}-D_{s 1}\left(\dot{x}_{1}-\dot{x}_{2}\right)-D_{s 2}\left(\dot{x}_{1}-\dot{x}_{2}\right)^{2}-f_{1}(x, t)+h(x, t) u\right]-\ddot{x}_{d}
\end{array} .\right.
$$

Note that the second relation of equation (1) is used to determine the value of $x_{2}$ in each time step. In an actual system, there is no need to solve such relations for determining $x_{2}$, because its values are directly obtained from the installed sensors. However, since this study is a simulation, the second relation in equation (1) must be solved. Moreover, the vertical variations of the road are considered as white Gaussian noise; this section briefly discusses the stochastic Itô and Stratonovich integrals.

The Itô and Stratonovich integrals are explained in the following given their application in solving stochastic differential equations. These two integrals are widely used to solve such equations in cases where the studied system is subjected to the Wiener process. Consider $\int_{0}^{T} h(t) \mathrm{d} w$, where 
$w:[0, T] \times \Omega \longrightarrow R$ represents a Wiener process and $X:$ $[0, T] \times \Omega \longrightarrow R$ is a semimartingale compatible with filtration. Under these conditions, and relying on the Riemann Sum, the above integral value can be expressed in two ways:

$$
\begin{gathered}
\sum_{j=0}^{N-1} h\left(t_{j}\right)\left[w\left(t_{j+1}\right)-w\left(t_{j}\right)\right], \\
\sum_{j=0}^{N-1} h\left(\frac{t_{j}+t_{j+1}}{2}\right)\left[w\left(t_{j+1}\right)-w\left(t_{j}\right)\right] .
\end{gathered}
$$

Equations (4) and (5) represent the integral forms of Itô and Stratonovich relations, respectively, where $N$ is the number of divisions in the time interval $[0, T]$ and $j$ is the counter. $\mathrm{d} t=T / N$ represents the time interval between two samples. It must be noted that the Wiener process is the integral of white noise; in other words, $x_{0} \mathrm{~d} t=\mathrm{d} w$. Since the term $K_{t 1} x_{0} \mathrm{~d} t$ represents the term $h(t) \mathrm{d} w$ and the value of $K_{t 1}$ is constant with respect to time, Itô and Stratonovich assume identical forms according to equations (4) and (5) [40].

Having discussed the Itô and Stratonovich integrals, the design of the sliding mode controller is explained in the following. Considering $e_{1}=x_{1}-x_{d}$ and $e_{2}=\dot{x}_{1}-\dot{x}_{d}$ and by placing $x_{1}=e_{1}+x_{d}$ and $\dot{x}_{1}=e_{2}+\dot{x}_{d}$ in equation (3), the dynamic error equation can be rewritten as follows:

$$
\left\{\begin{array}{l}
\dot{e}_{1}=e_{2}, \\
\dot{e}_{2}=\frac{1}{M_{1}}\left[-K_{s 1}\left(e_{1}+x_{n}\right)-K_{s 2}\left(e_{1}+x_{n}\right)^{3}-D_{s 1}\left(\dot{e}_{1}+\dot{x}_{n}\right)-D_{s 2}\left(\dot{e}_{1}+\dot{x}_{n}\right)^{2}-f_{1}(e, x, t)+h(e, x, t) u\right]-\ddot{x}_{d}
\end{array}\right.
$$

where $x_{n}=x_{d}-x_{2}$ and $\dot{x}_{n}=\dot{x}_{d}-\dot{x}_{2}$.

To design the sliding mode controller, we consider the sliding surface $s$ and its time derivative as follows:

$$
\begin{aligned}
& s=e_{2}+\lambda e_{1}, \\
& \dot{s}=\dot{e}_{2}+\lambda \dot{e}_{1}=\dot{e}_{2}+\lambda e_{2} .
\end{aligned}
$$

By inserting $e_{2}$ and $\dot{e}_{2}$ from equation (6) into (7), we have

$$
\dot{s}=\frac{1}{M_{1}}\left[-K_{s 1}\left(e_{1}+x_{n}\right)-K_{s 2}\left(e_{1}+x_{n}\right)^{3}-D_{s 1}\left(\dot{e}_{1}+\dot{x}_{n}\right)-D_{s 2}\left(\dot{e}_{1}+\dot{x}_{n}\right)^{2}-f_{1}(e, x, t)+h(e, x, t) u\right]-\ddot{x}_{d}+\lambda e_{2}
$$

\subsubsection{Lyapunov Function and Controller Stabilization:} In this section, we shall discuss the Lyapunov function and how to stabilize the controller using the time derivative of the Lyapunov function [41-43].

Definition 1. The Lyapunov point $\bar{x}$ is considered stable if there exists $\varepsilon>0$ such that $\delta(\varepsilon)>0$ and $\|x(0)-\bar{x}\|<\delta$; then, $\|x(t)-\bar{x}\|<\varepsilon$ for all $t>0$. Note that the initial condition $\delta(\varepsilon)$ must exist for $\varepsilon>0$.

Definition 2. The fixed point $\bar{x}$ is asymptotically stable if it is stable and there exists $\eta>0$ where $\lim _{t \rightarrow \infty}\|x(t)-\bar{x}\|=0$ whenever $\|x(0)-\bar{x}\|<\eta$.

The Lyapunov stability and asymptotic stability are shown in Figures 2(a) and 2(b), respectively. As shown in Figure 2(a)), in Lyapunov's stability, $\|x(t)-\bar{x}\|$ lies in the surrounded space and remains in this region for different times. Note that, in this case, the expression $\|x(t)-\bar{x}\|$ is not necessarily required to reach zero as time approaches $\infty$. However, as shown in Figure $2 \mathrm{~b}$ ), $x_{1}(t)$ moves toward $\bar{x}$ over time, and as $t \longrightarrow \infty$, the value of $\|x(t)-\bar{x}\|$ approaches zero.

For Lyapunov stability, $\delta(\varepsilon)$ actually represents the radius of basin attraction set $\bar{x}$. The aforementioned concepts help better understand the design and function of the sliding mode controller. In the absence of uncertainty, noise, and disturbance, asymptomatic stability is achievable for deterministic systems using sliding mode controllers. However, these factors are inherently present in dynamic systems, making it impossible to have asymptotic stability, and merely Lyapunov stability would be achievable. In deterministic systems, we can implement all the specifications accurately to ensure asymptotic stability, and even the convergence rate of the dynamic error can be specified. However, in presence of uncertainty or noise in the system, asymptomatic stability cannot be guaranteed. As shown in equations (1) and (8), the values for parameters of $h(x, t), f_{1}(x, t), f_{2}(x, t)$, and $x_{0}$ are unpredictable and thus cannot be accurately modeled in the controller. Therefore, asymptomatic stability for this system may not be guaranteed, and only the Lyapunov stability can be achieved.

In the following, a definition is given for Lyapunov's function that its existence implies the existence of system stability. Naturally, it should be noted that the lack of such function is not a reason for instability. Actually, the existence of such a term is a sufficient condition for stability. Although this definition may not provide a straightforward method for determining the control law, but based on this definition, the control law will be presented to stabilize the feedback control system.

Definition 3. Consider the energy function or Lyapunov function $V$ such that $V>0$. If $\dot{V}<0$, then the stability of this system is guaranteed. 


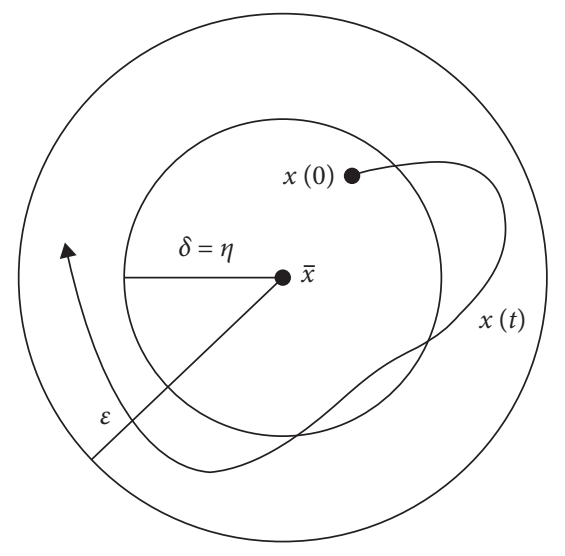

(a)

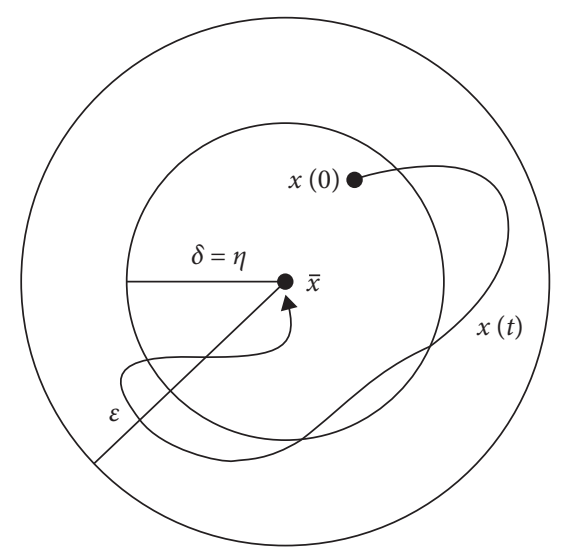

(b)

FIgURE 2: (a) Lyapunov stability and (b) asymptotic stability [44].

In fact, $\dot{V}<0$ indicates the decrescent rate of the energy function, which indicates a decrease in energy and tendency of $V$ toward zero (for $\dot{V}>0$, the energy of the system gradually increases and the system goes toward instability). In the continuation of this section, according to the above definition, we will determine the control law for the feedback control system with the Lyapunov stability approach.
The considered energy function is $V=(1 / 2) s^{2}$, which represents a positive function. The time derivative of this function is equal to $\dot{V}=s \dot{s}$. Now, if we can satisfy the condition $\dot{V}=s \dot{s}<0$, the control feedback rule may be presented according to Definition 3.

By inserting $\dot{s}$ from equation (8) in $\dot{V}=s \dot{s}$, we have

$$
\dot{V}=s\left\{\frac{1}{M_{1}}\left[-K_{s 1}\left(e_{1}+x_{n}\right)-K_{s 2}\left(e_{1}+x_{n}\right)^{3}-D_{s 1}\left(\dot{e}_{1}+\dot{x}_{n}\right)-D_{s 2}\left(\dot{e}_{1}+\dot{x}_{n}\right)^{2}-f_{1}(x, t)+h(x, t) u\right]-\ddot{x}_{d}+\lambda e_{2}\right\} .
$$

Now for stability, we have to determine $u$ in such a way that the above equation remains negative.

Determining $u$ through this relationship is complex. Therefore, in the following, we define an upper limit for $\dot{V}$. If the seminegative condition for this upper limit is met, then the condition $\dot{V}<0$ is also met. To determine $u$, we assume that the whole expression to the right of this function is equal to $-\eta h(x, t)|s|$ where $\eta$ is a positive number. According to $\eta>0, h(x, t)>0$, and $|s|>0,-\eta h(x, t)|s|$ and $\dot{V}$ become negative and the condition of stable feedback control is guaranteed. In fact, this equation is the basis for determining control law and all calculations made in equations (12) and (13) are to determine this law:

$$
\begin{aligned}
\dot{V} & \leq \frac{|s| h(x, t)}{M_{1}}\left\{\frac{1}{h_{0}}\left[K_{s 1}\left|e_{1}+x_{n}\right|+K_{s 2}\left|e_{1}+x_{n}\right|^{3}+D_{s 1}\left|\dot{e}_{1}+\dot{x}_{n}\right|+D_{s 2}\left(\dot{e}_{1}+\dot{x}_{n}\right)^{2}+F_{1}+\frac{\left|\ddot{x}_{d}\right|}{M_{1}}+\frac{\lambda}{M_{1}}\left|e_{2}\right|\right]\right\} \\
+\frac{s h(x, t) u}{M_{1}} & =-\eta h(x, t)|s| \\
\dot{V} & \leq-\eta h(x, t)|s|,
\end{aligned}
$$

where $F_{1}=\sup \left\{\left|f_{1}(x, t)\right|\right\}$ and $h(x, t) \geq h_{0}>0$ and $\eta$ represents a positive number. The positive function $F$ can then be defined as follows:

$$
F=\frac{1}{M_{1} h_{0}}\left[K_{s 1}\left|e_{1}+x_{n}\right|+K_{s 2}\left|e_{1}+x_{n}\right|^{3}+D_{s 1}\left|\dot{e}_{1}+\dot{x}_{n}\right|+D_{s 2}\left(\dot{e}_{1}+\dot{x}_{n}\right)^{2}+F_{1}+\frac{\left|\ddot{x}_{d}\right|}{M_{1}}+\frac{\lambda}{M_{1}}\left|e_{2}\right|\right] .
$$


Inequality (10) can be rewritten as follows:

$$
\dot{V} \leq|s| F h(x, t)+s \frac{h(x, t) u}{M_{1}}=-|s| \eta h(x, t) .
$$

Considering the right side of (12) and dividing it by $h(x, t)$, the following relation is obtained:

$$
\begin{aligned}
s \frac{u}{M_{1}} & =-|s| \eta-|s| F, \\
u & =-M_{1}(\eta+F) \frac{|s|}{s}=-M_{1}(\eta+F) \operatorname{sign}(s) .
\end{aligned}
$$

The above equation indicates the control rule obtained based on the sliding mode method, where the function $\operatorname{sign}(s)$ is defined as follows:

$$
\operatorname{sign}(s)= \begin{cases}1, & s>0 \\ 0, & s=0 \\ -1 & s<0\end{cases}
$$

Equations (11) and (13) together with the relation of $s=$ $\dot{e}_{1}+\lambda e_{1}$ are the main blocks to design the sliding mode controller. To simplify the feedback process and the basis for determining the control law; the method of determining control law and the control process is presented in Figure 3. As it turns out, the most complex part of control design is determining the function of $F$. To determine this term in each simulation and in each time step, the values of $x_{1}, x_{2}$, $\dot{x}_{1}$, and $\dot{x}_{2}$ are taken from the car model (using sensors in the real system). Also, $\dot{x}_{d}$ and $\ddot{x}_{d}$ are obtained based on reference input or $x_{d}$. Also, the sliding surface of $s$ can be obtained using blocks of $\lambda e_{1}$ and $\mathrm{d} e_{1} / \mathrm{d} t$. Finally, according to the sign of sliding surface and using $-M_{1} F \operatorname{sign}(s)$ and $-M_{1} \eta \operatorname{sign}(s)$, the control force is determined, and it is imposed to the suspension system. As it is known, the design terms consist of $\lambda$ and $\eta$ where they should be determined by the designer. Another factor that is very important in the dynamic behavior of the system is uncertainty. There is also another parameter of $\sigma$ that will be discussed later, which will be used in the form of $s /(|s|+\sigma)$ and instead of $\operatorname{sign}(s)$ to reduce the chatting phenomenon. Therefore, in the results section, the effects of these parameters on the dynamic behavior of the suspension system will be fully investigated.

In the continuation of this part, the effects of controller parameters of convergence speed of controller will be discussed. If the error is considered as $\tilde{x}=x-x_{d}$, the sliding surface equation can be reflected in the form of $s(x, t)=((\mathrm{d} / \mathrm{d} t)+\lambda)^{n-1} \tilde{x}$. In this article $n=2$. Also for $n=3$, the sliding surface is presented in the form of $s=\tilde{x}+2 \lambda \tilde{x}+\lambda^{2} \tilde{x}$. Given the initial conditions $\tilde{x}(0)=e(0)$, the equation for the sliding surface represents a linear differential equation with constant coefficients. Based on [45], the tracking error tends to zero exponentially and with a time constant of $(n-1) / \lambda$, where for $n=2$, it is equal to $1 / \lambda$. This time constant is for the case where the initial error is placed on the sliding surface. If the initial position of the error is not on the sliding surface, it reaches the surface in less than $|s(t=0)| / \eta$ and then starts sliding on this surface. Therefore, the parameters of $\lambda$ and $\eta$ are the most important parameters in the study of convergence speed and their effect on the dynamic behavior of the system will be addressed in the continuation of this work.

Of course, the important point is that, due to the existence of white Gaussian noise, the tracking error or the position of the system will never be settled at zero. This state is like an example of a forced vibration with stochastic nature.

In the above section, the design method of a sliding mode controller was explained based on the Lyapunov function. In the following section, some notes are discussed on designing a sliding mode controller for a dynamic system that is only affected by white noise. Consider the sliding mode surface as follows [46]:

$$
\dot{s}=M(x, t)+B(x, t) u+H(x, t) \dot{v}
$$

where $\dot{v}$ expresses the Wiener process. Under these conditions, instead of the above equations, the definition of the Lyapunov function can be alternatively used for a stochastic system as the range of the basin attraction set can be more conveniently determined. Note that in this case, we could consider the Lyapunov function as $V=(1 / 2) E\left[s^{2}\right]$ instead of $V=(1 / 2) s^{2}$, where $E[\bullet]$ indicates the expected value. In this case, instead of $\dot{V}$, we must use $\mathrm{d} V=(1 / 2) E\left[\mathrm{~d}\left(s^{2}\right)\right]$, where $\mathrm{d} V$ represents infinitesimal variations in $V$. Moreover, contrary to the case considered in this study assuming $(\mathrm{d} / \mathrm{d} t)\left[(1 / 2)\left(s^{2}\right)\right]=2 s \dot{s}$, in the new situation, the following equation based on Ito's definition of derivation must be used for determining the infinitesimal changes in $s^{2}$ :

$$
\mathrm{d}\left(s^{2}\right)=2 s \mathrm{~d} s+\mathrm{d} s \mathrm{~d} s
$$

Further information can be found in [47, 48]. Considering that no information is available on the expected values $E\left[x_{2}\right]$ and $E\left[\dot{x}_{2}\right]$, this method may no longer be used to design a sliding mode controller, since $x_{2}$ and $\dot{x}_{2}$ are not necessarily a random Wiener process or white noise.

At the end of this section and before presenting the results, we are interested in presenting the importance of conservatism in controller design. One of the important topics in designing a controller based on the definition of Lyapunov function is conservatism in the presence of uncertainties. Reducing such a factor can increase the quality of system control or decrease the controller force. Considerable studies have been done in this field, which can be stated as the Linear Matrix Inequality (LMI) and parameter-dependent Lyapunov function methods [49]. The use of the LMI method to reduce the conservatism of linear systems is presented in [50]. One of the key features of the LMI method in reducing conservatism is its ability to be generalized to nonlinear systems and its easy implementation in MATLAB software [51]. Another study that has been done in this field is based on the quadratic Lyapunov function [49]. Other investigations in this case and based on the definition of Lyapunov function are presented in [49]. In this study, due to the presence of uncertainty, reducing the conservative can improve the controller design or decrease the control force. However, due to the fact that the study of this topic in this paper 


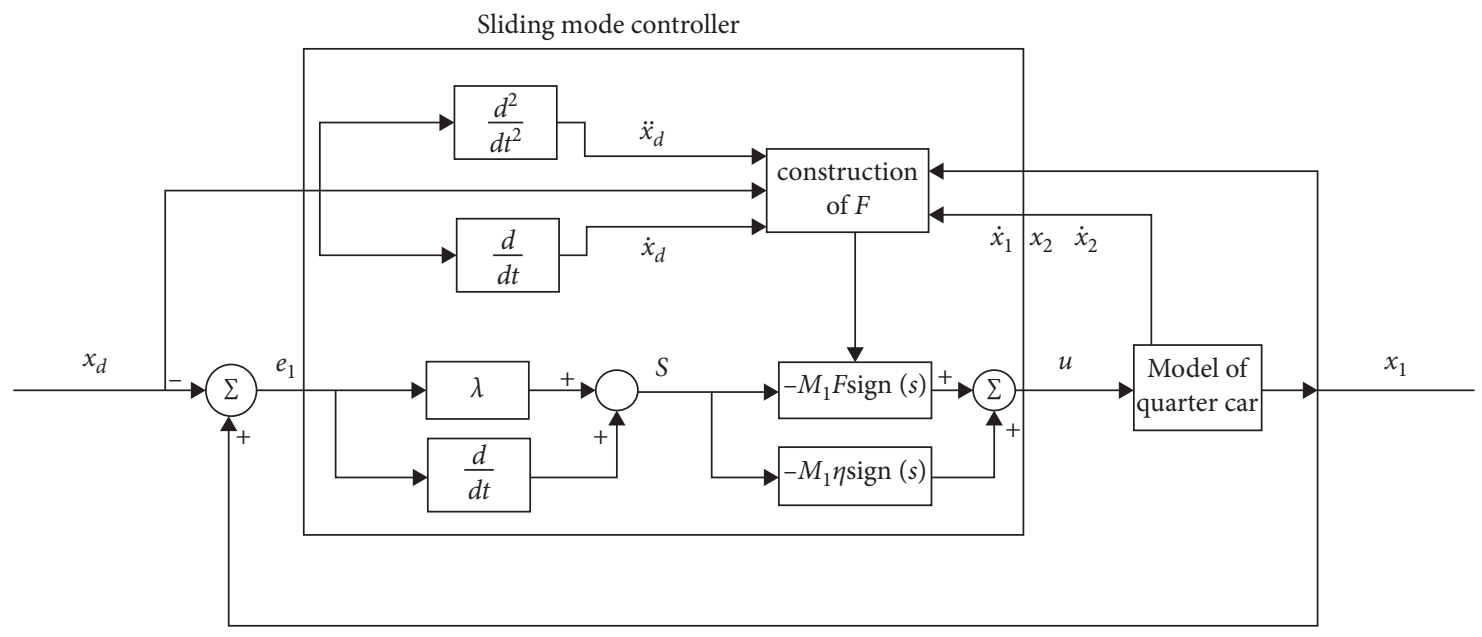

Figure 3: Block diagram of determining feedback control.

TABle 1: Suspension parameters [51].

\begin{tabular}{lc}
\hline Suspension parameters & Value \\
\hline Sprung mass $M_{1}$ & $295 \mathrm{~kg}$ \\
Unsprung mass $M_{2}$ & $39 \mathrm{~kg}$ \\
Linear damping coefficient $D_{s 1}$ & $3482 \mathrm{Ns} / \mathrm{m}$ \\
Nonlinear square damping coefficient $D_{s 2}$ & $580 \mathrm{Ns} / \mathrm{m}^{2}$ \\
Linear spring stiffness coefficient $K_{s 1}$ & $15302 \mathrm{~N} / \mathrm{m}$ \\
Nonlinear square spring stiffness coefficient $K_{s 2}$ & $2728 \mathrm{~N} / \mathrm{m}$ \\
Linear tire stiffness coefficient $K_{t 1}$ & $60063 \mathrm{~N} / \mathrm{m}$ \\
Nonlinear square tire stiffness coefficient $K_{t 2}$ & $42509 \mathrm{~N} / \mathrm{m}$ \\
Nonlinear cube tire stiffness coefficient $K_{t 3}$ & $22875 \mathrm{~N} / \mathrm{m}$
\end{tabular}

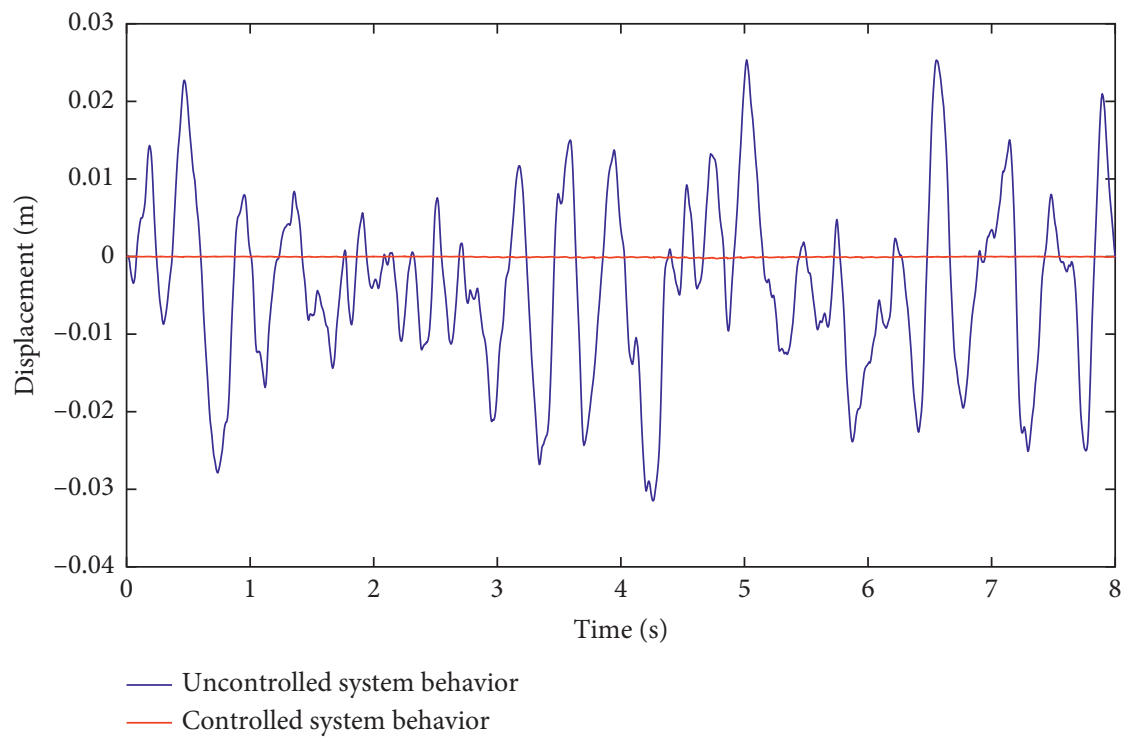

FiguRE 4: Uncontrolled and controlled system behavior using designed sliding mode controller for $\lambda=1$ and $\eta=10$.

causes much complexity and increases the unconventional scope of the paper, it is generally referred to here. Due to the significant importance of reducing conservatism, this issue should be studied in future studies for the design of controllers in nonlinear dynamic systems in the presence of white Gaussian noise.

\section{Results and Discussions}

4.1. Control ofQuarter-CarSystem. In this section, we intend to present and discuss the extracted results. The specifications of the studied system are presented in Table 1 according to the ISO 2631-1:1997 standard [51]. To 


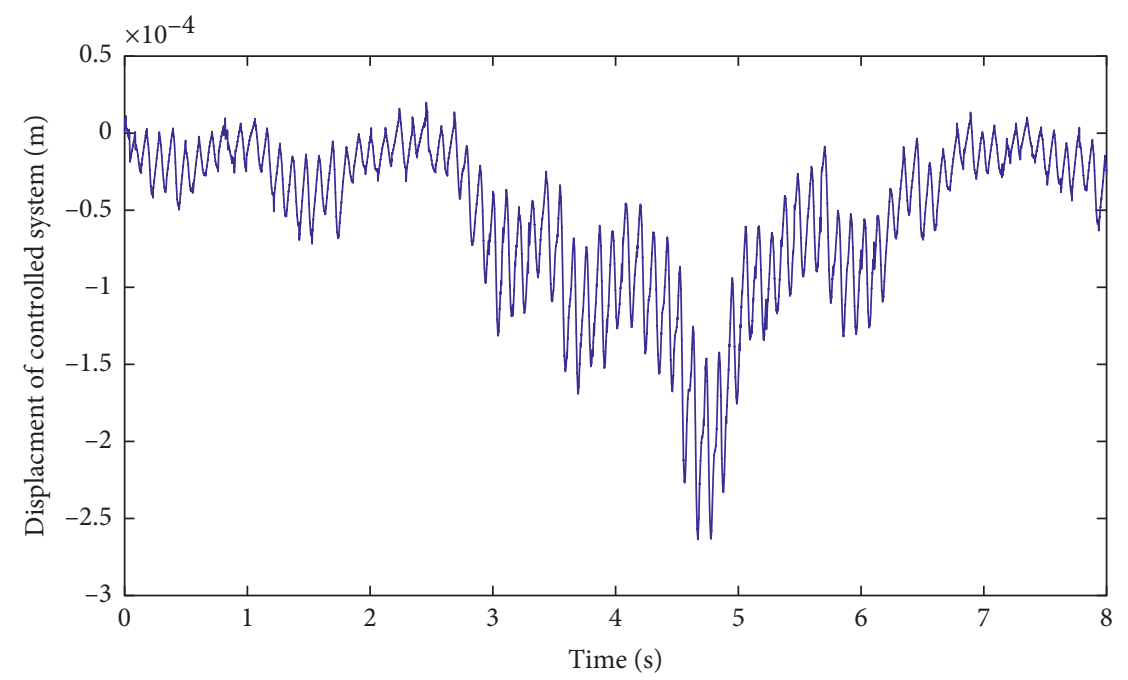

FIGURE 5: Controlled system behavior using designed sliding mode controller for $\lambda=1$ and $\eta=10$.

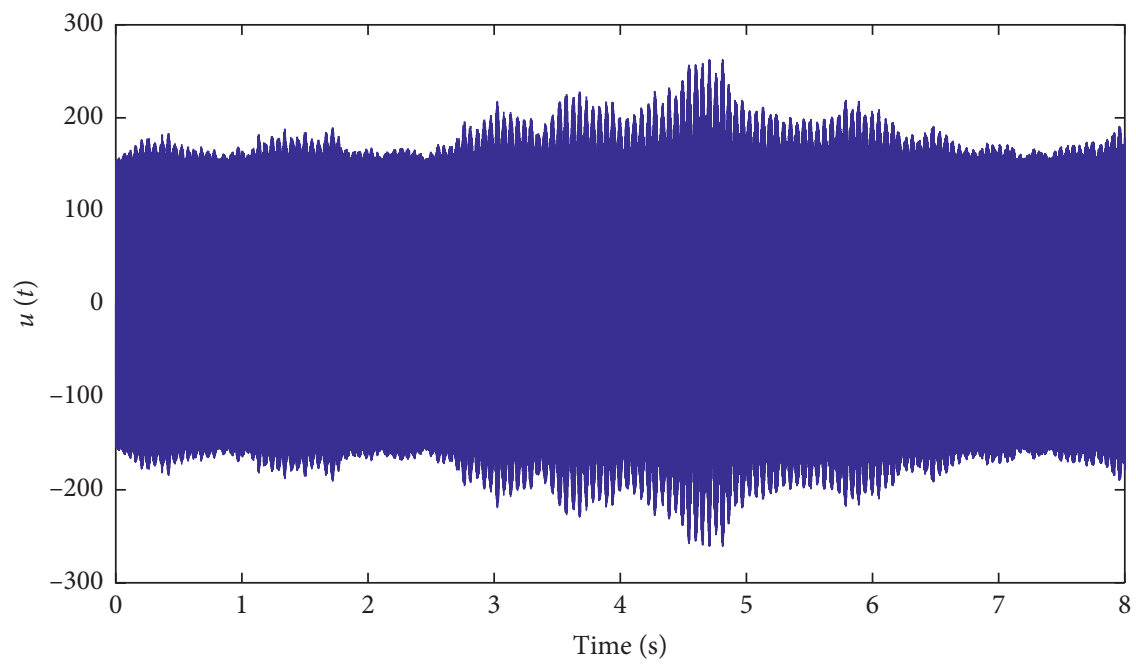

(a)

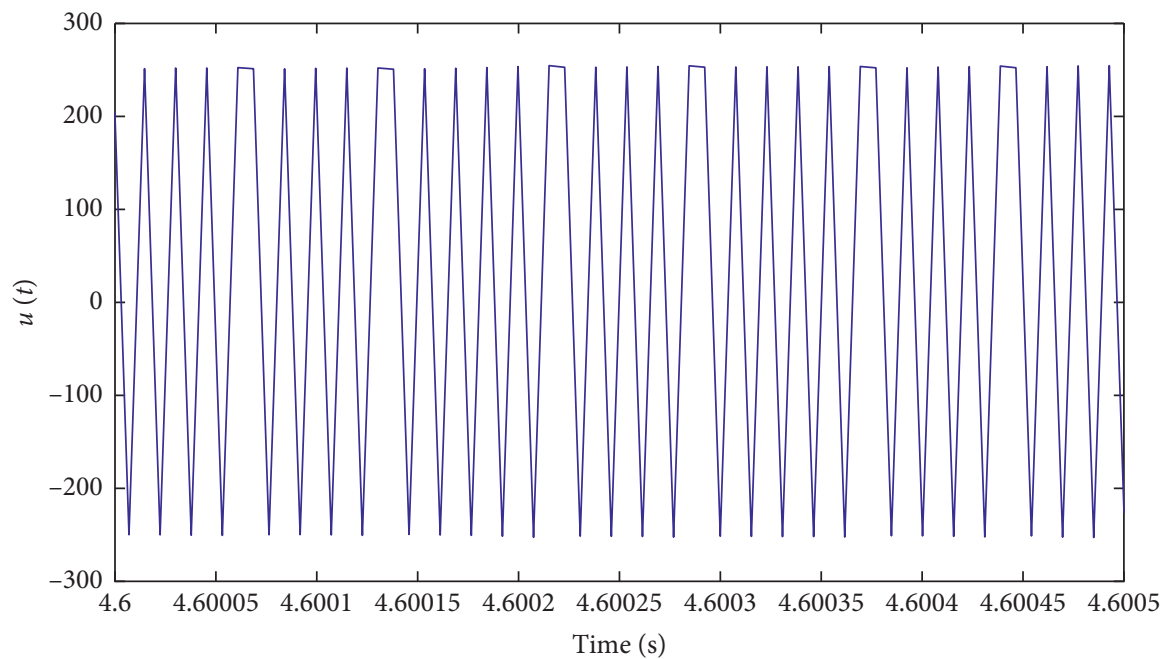

(b)

Figure 6: Variation of a control parameter of $u(t)$ for Figure 5. (a). $u(t)$. (b) Detailed view of Figure 6(a). 


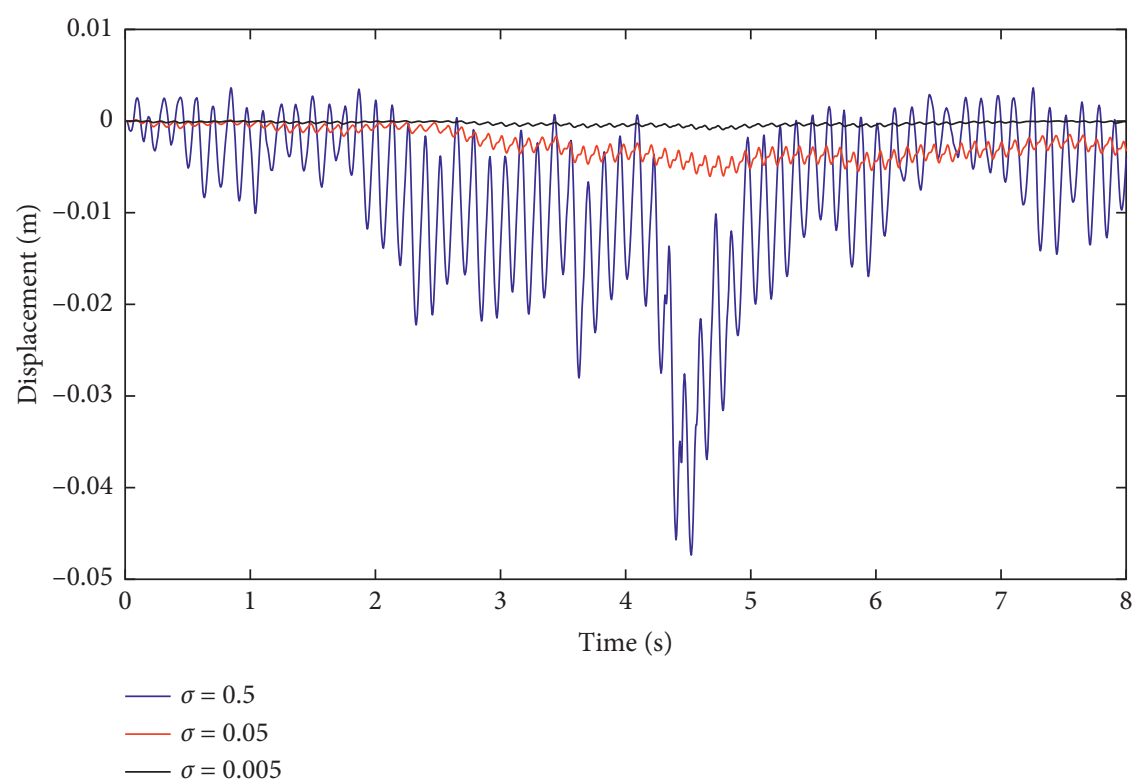

(a)

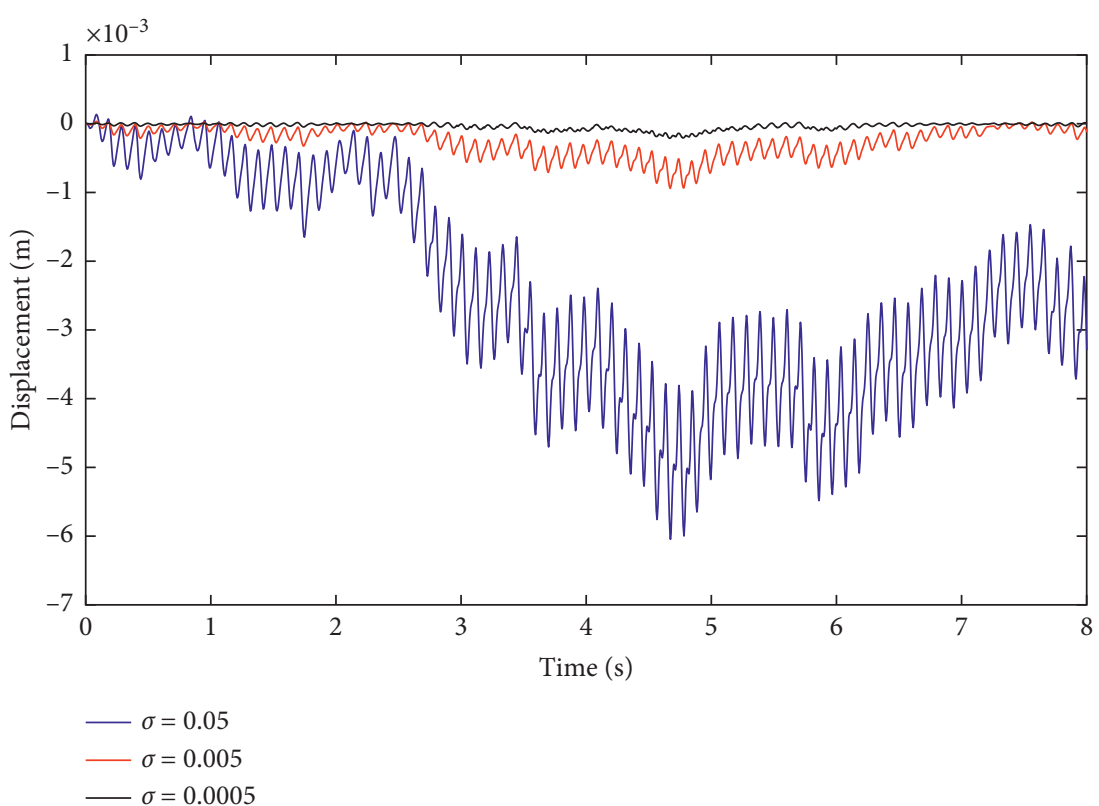

(b)

FIgURE 7: Controlled system behavior for different magnitudes of $\sigma$ and $\lambda=1, \eta=10$. (a) (b)

TABLE 2: Maximum displacement versus parameter of $\sigma$.

\begin{tabular}{lcccc}
\hline$\sigma$ & 0.5 & 0.05 & 0.005 & 0.0005 \\
\hline Maximum displacement & 0.047 & 0.006 & 0.00094 & 0.00021 \\
\hline
\end{tabular}

obtain the results, $x_{d}$ is considered as a constant with zero value.

Figure 4 shows the behavior of vertical movement of mass $M_{1}$ in the controlled and uncontrolled cases for $\lambda=1$ and $\eta=10$. As shown in the figure, the use of a sliding mode controller significantly reduces the amplitude of the vibration. Furthermore, the behavior of the active system is negligible compared to the passive system. Figure 5 shows only the behavior of the active system.

As mentioned, the chattering phenomenon is a major problem of the sliding mode controller. Given the function $\operatorname{sign}(s)$ in the control force equation, the amount of this 


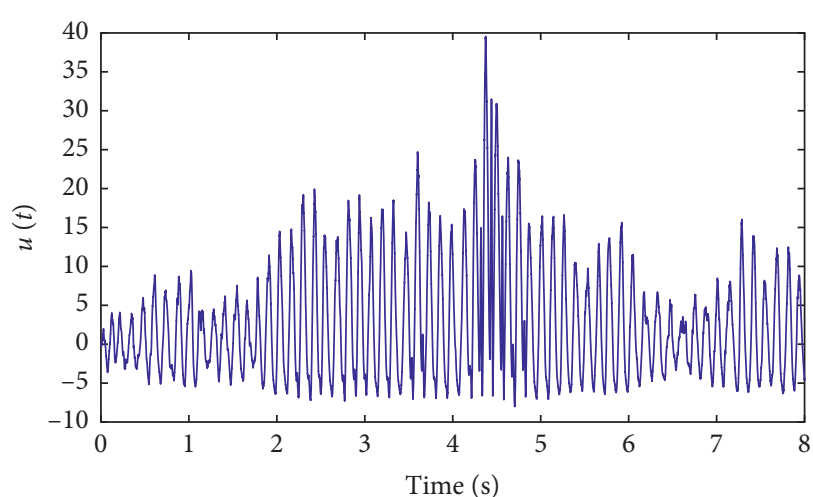

(a)

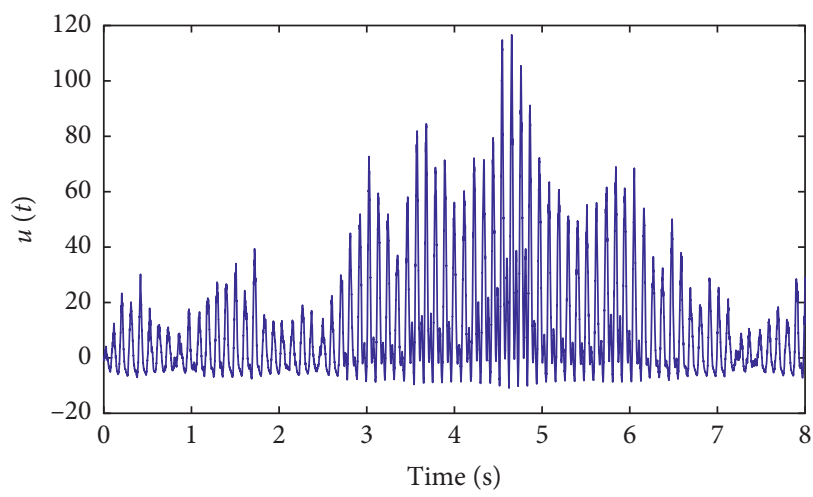

(c)

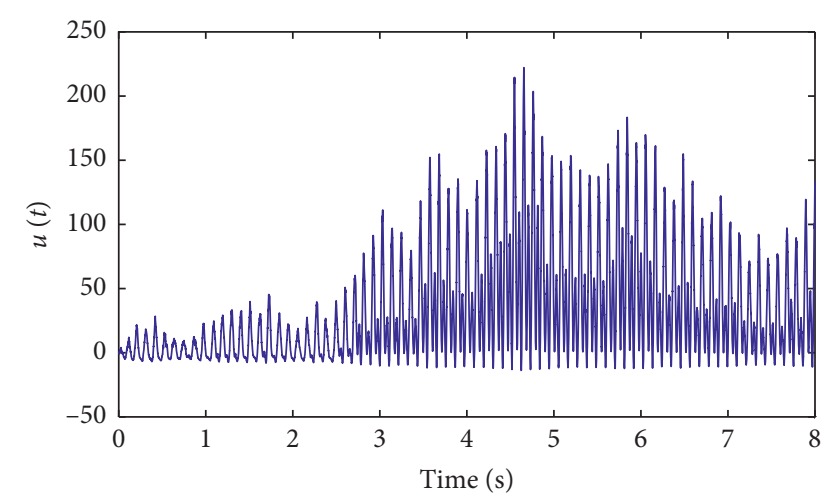

(b)

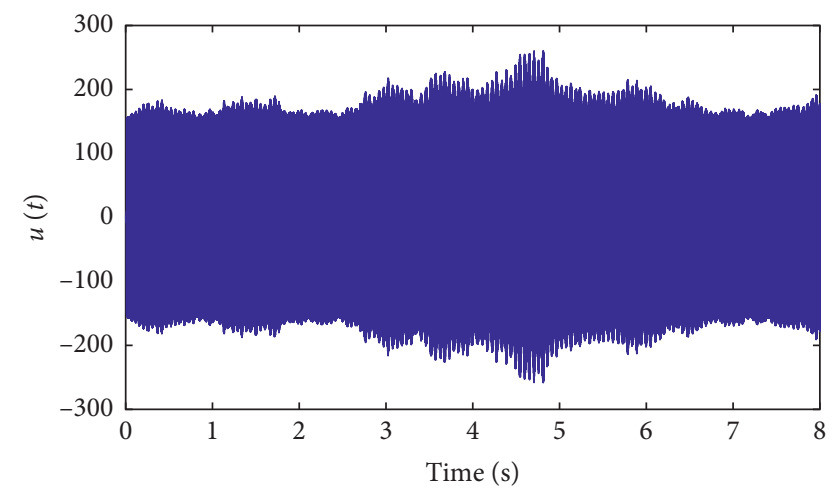

(d)

Figure 8: Variation of the control parameter of $u(t)$ for Figure 7. (a). $\sigma=0.5$, (b). $\sigma=0.05$, (c). $\sigma=0.005$, and (d). $\sigma=0.0005$.

TABLE 3: Maximum amount of control force $u(t)$ for various amount of $\sigma$.

\begin{tabular}{lcccc}
\hline$\sigma$ & 0.5 & 0.05 & 0.005 & 0.0005 \\
\hline Maximum amount of $u(t)$ & 39.55 & 222.10 & 116.61 & 260.62 \\
\hline
\end{tabular}

force changes its sign at a high frequency, which is due to the fact that $\operatorname{sign}(s)$ is discontinuous at $s=0$. The changes in the control force presented in Figure 5 are depicted in Figure 6. As shown, the control force changes sign at a high frequency.

Note that the chattering phenomenon damages the actuators and reduces its lifecycle. Therefore, most designs of the sliding mode controller try to somehow eliminate the chattering phenomenon. As mentioned before, the function $\operatorname{sign}(s)$ is responsible for the chattering phenomenon because of discontinuity at point zero. To eliminate chattering, this article uses $s /(|s|+\sigma)$ instead of $\operatorname{sign}(s)$, where $\sigma$ is a positive and small number mathematically defined as follows:

$$
\varphi_{\sigma}(s)= \begin{cases}1, & |s|>\sigma \\ \sigma^{-1} s, & |s| \leq \sigma\end{cases}
$$

The function $\varphi_{\sigma}(s)$ is an approximation of $\operatorname{sign}(s)$ and is not its exact equivalent. The accuracy of this approximation is increased for smaller $\sigma$ values.

Figure 7 shows the results of chattering reduction for different values of $\sigma$.
The maximum displacements shown in Figure 7 are presented in Table 2. As can be seen, with a decrease of $\sigma$, the level of maximum displacement decreases.

Moreover, variations of control forces for different values of $\sigma$ are presented in Figure 8. As shown in Figure 7, by decreasing $\sigma$, the vibration amplitude is decreased. As shown in Figures $8(\mathrm{a})-8(\mathrm{c})$, the use of the function $\varphi_{\sigma}(s)$ eliminates the chattering phenomenon. However, as shown in Figure $8(\mathrm{~d})$, the chattering phenomenon is still present for $\sigma=0.0005$, suggesting that chattering cannot be eliminated for very small $\sigma$ values. Therefore, for proper operation of the controller and reduction of the vibrations, the values $\sigma=$ 0.05 and $\sigma=0.005$ are suitable, as they reduce the vibration amplitude and eliminate the chattering phenomenon. Note that, however, for $\sigma=0.5$, chattering is not detected, and according to Figure $7(\mathrm{a})$, the amplitude of vibrations is significant in this situation.

Also, the maximum amount of control force $u(t)$ for various amounts of $\sigma$ is presented in Table 3. As it can be seen from this table, the maximum amount of $u(t)$ increases with the decrease of $\sigma$.

In this section, we shall discuss the impact of changes in $\lambda$ and $\eta$ on the behavior of the system. Figure 9 shows the behavior of the system for various $\lambda$ values when $\eta=10$. As it can be seen, the maximum amplitude of the system behavior decreases as $\lambda$ increases.

Also, the maximum amount of displacement in terms of land is shown in Table 4. As can be seen from this table, the 


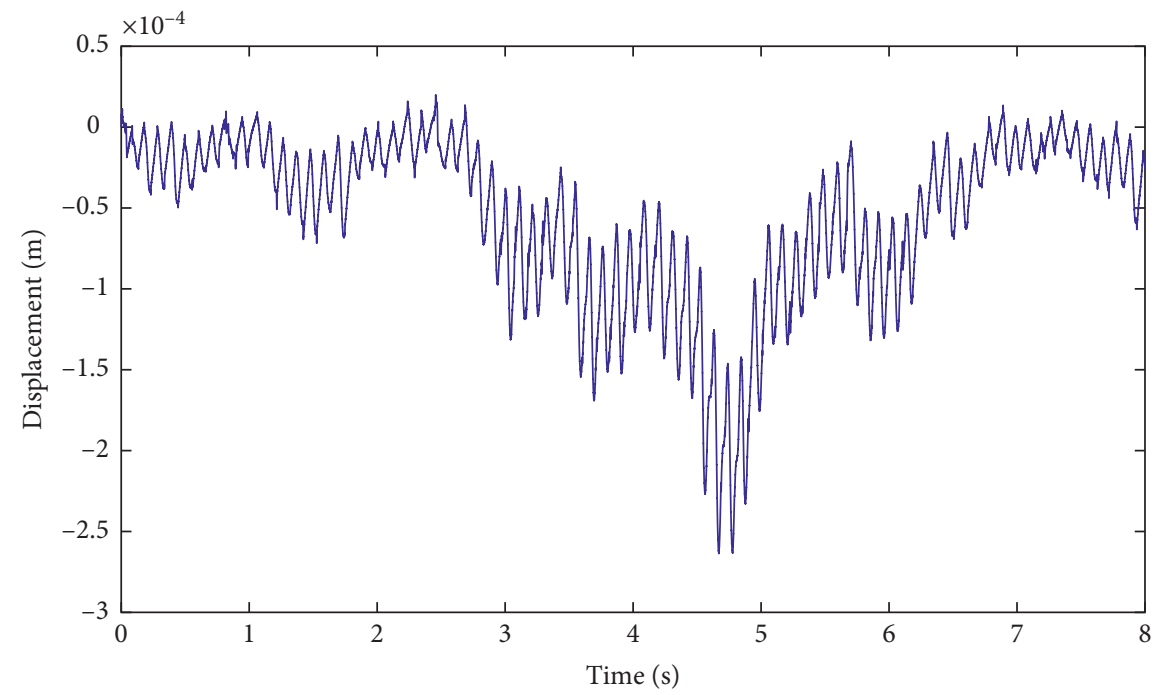

(a)

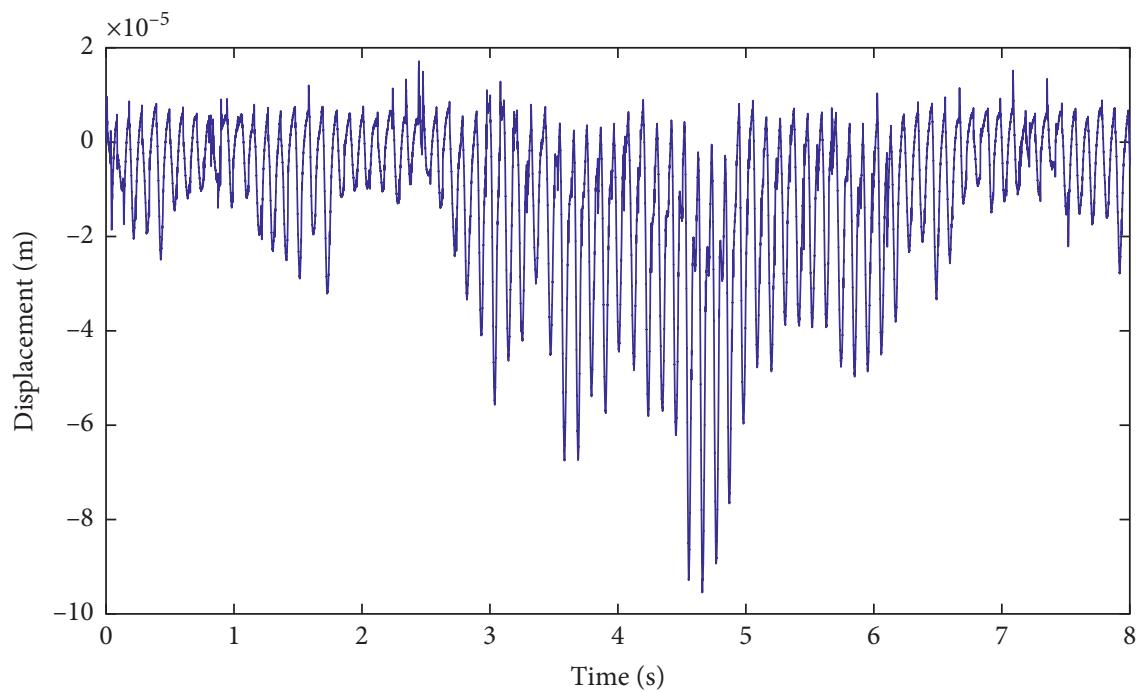

(b)

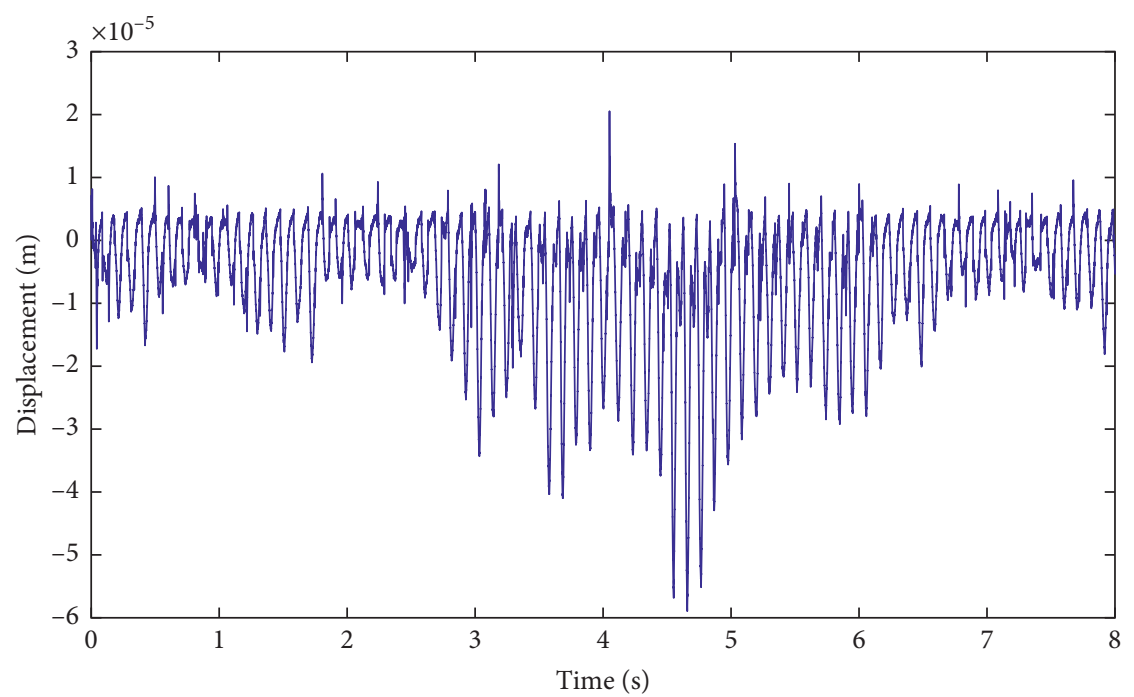

(c)

FIgUre 9: Continued. 


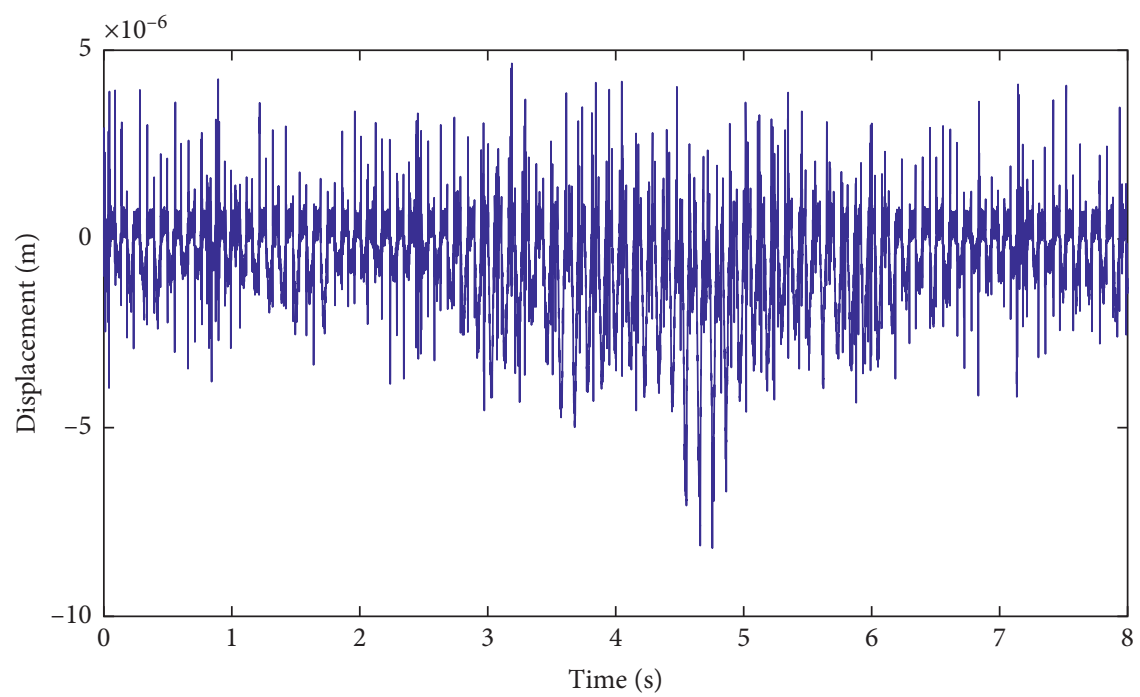

(d)

Figure 9: Controlled system behavior for different magnitudes of $\lambda$ and $\eta=10$. (a) $\lambda=1$, (b). $\lambda=5$, (c). $\lambda=10$, and (d). $\lambda=100$

TABLe 4: Maximum displacement versus $\lambda$.

\begin{tabular}{|c|c|c|c|c|c|}
\hline$\lambda$ & 1 & 2 & 5 & 10 & 100 \\
\hline Maximum displacement & 0.000263 & 0.000167 & $9.4 \times 10^{-5}$ & $5.89 \times 10^{-5}$ & $8.19 \times 10^{-6}$ \\
\hline
\end{tabular}

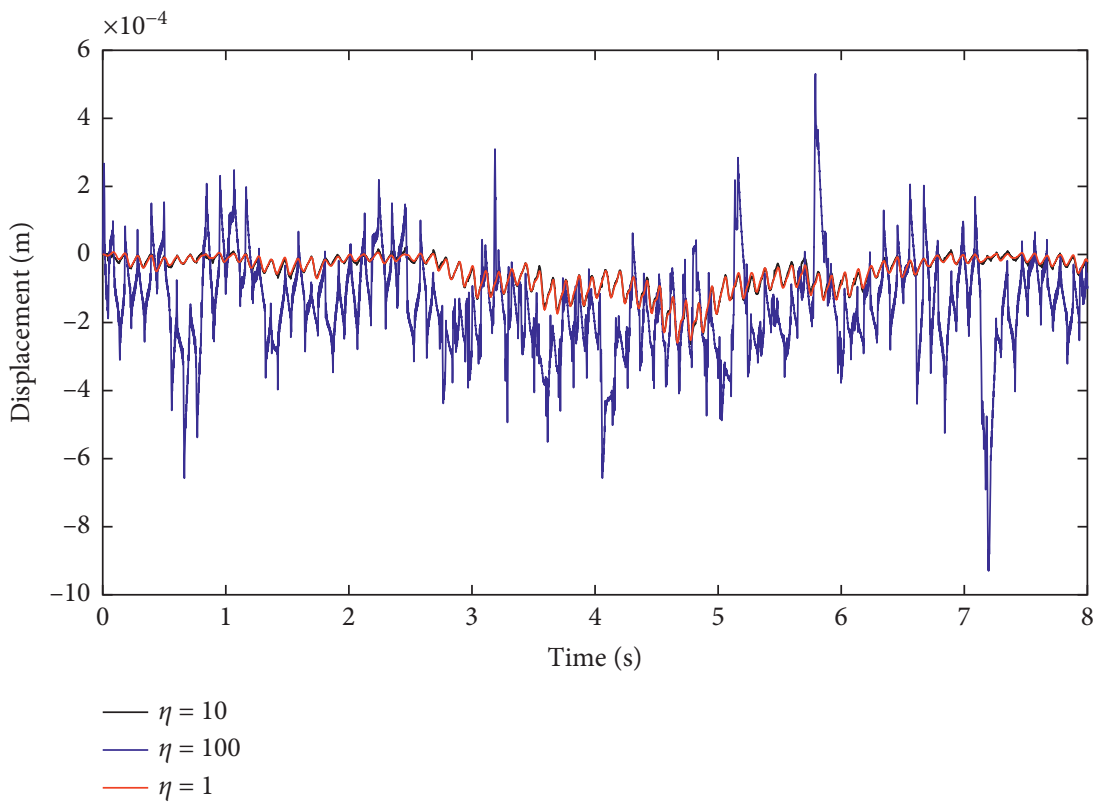

(a)

Figure 10: Continued. 


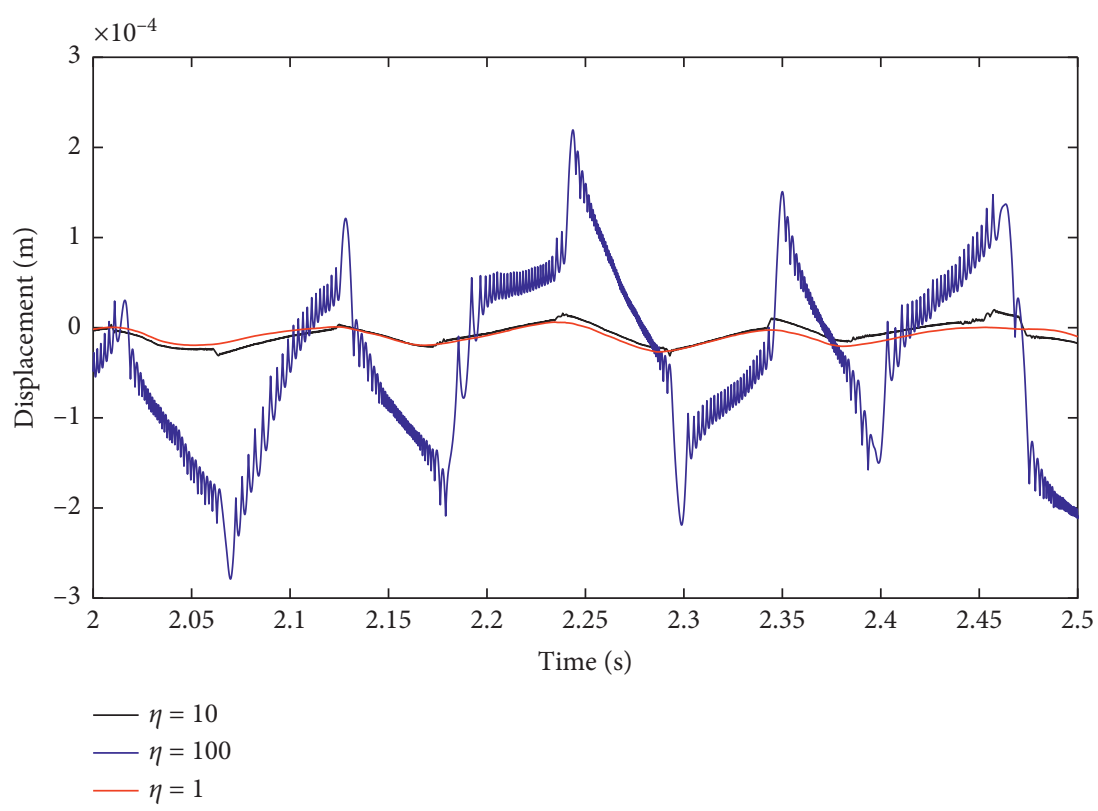

(b)

Figure 10: Controlled system behavior for different magnitudes of $\eta$ and $\lambda=1$. (a) (b). Detailed view of2 (a).

TABLe 5: Maximum displacement versus $\eta$.

\begin{tabular}{lccc}
\hline$\eta$ & 1 & 10 & 100 \\
\hline Maximum displacement & 0.000258 & 0.000263 & 0.000923 \\
\hline
\end{tabular}

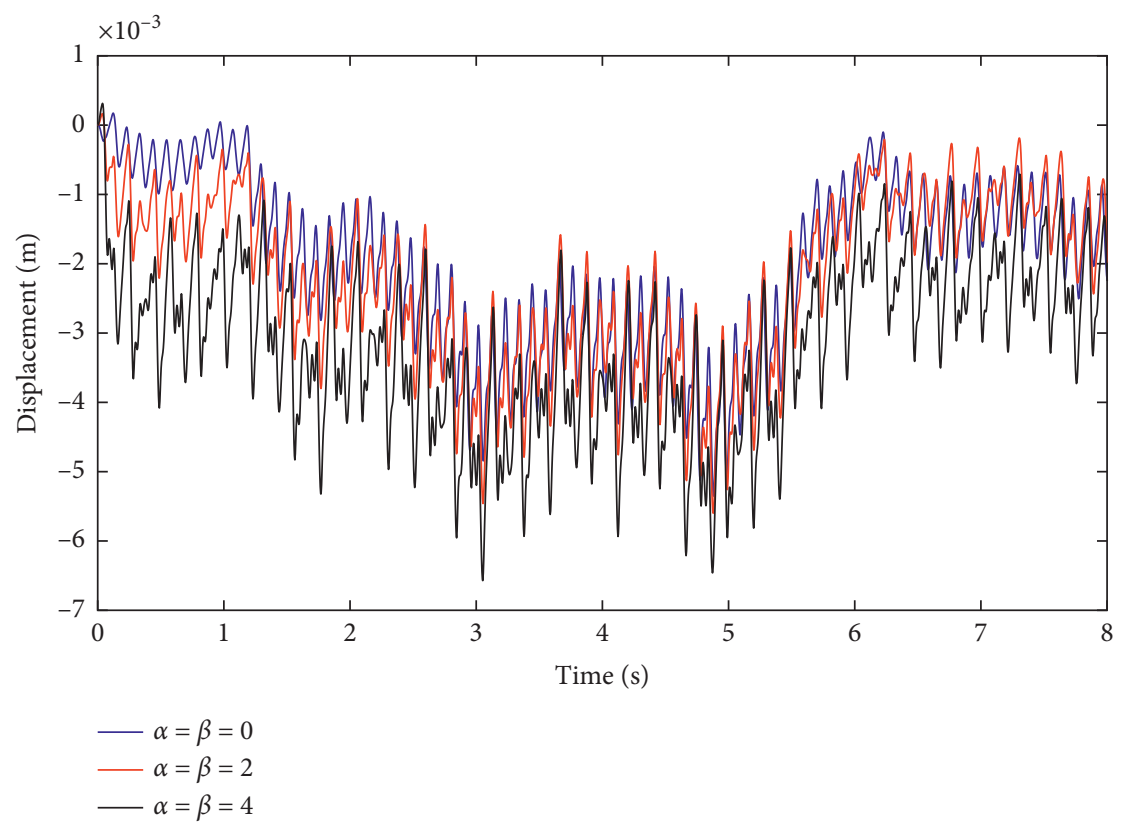

(a)

Figure 11: Continued. 


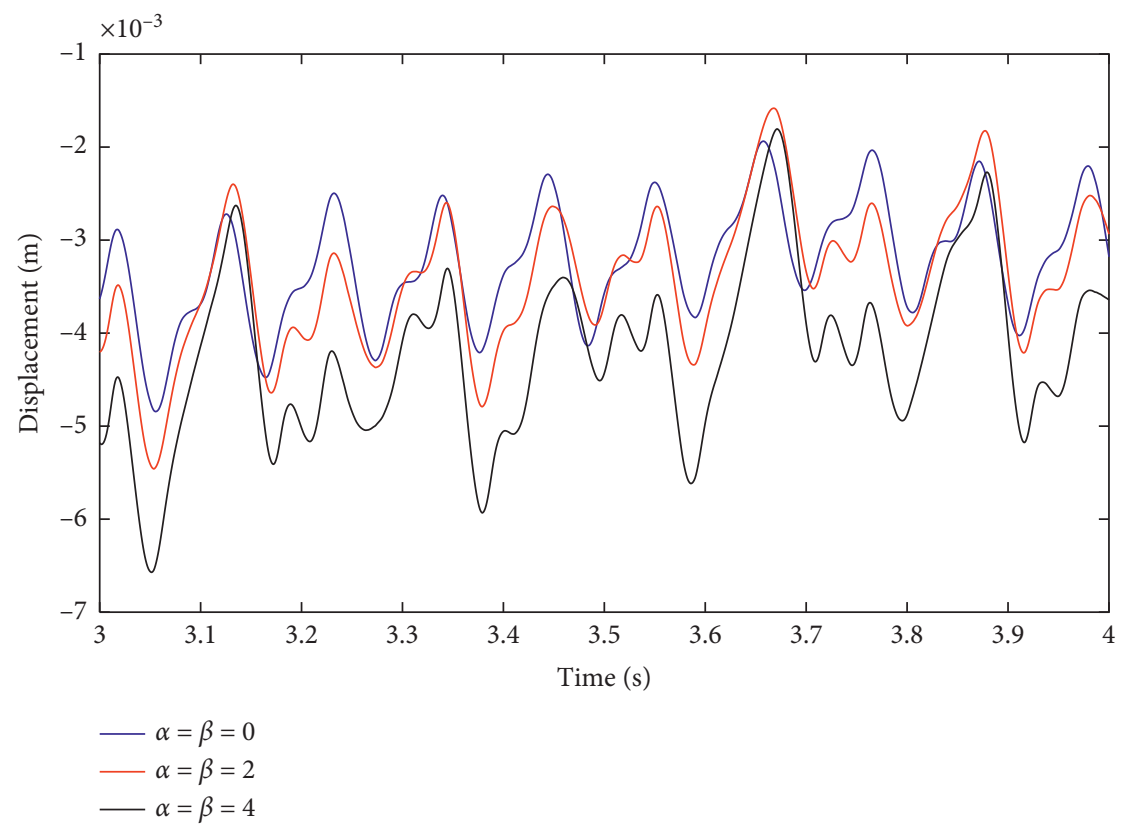

(b)

Figure 11: Controlled system behavior in the presence of system uncertainties. (a), (b). Detailed view of (a).

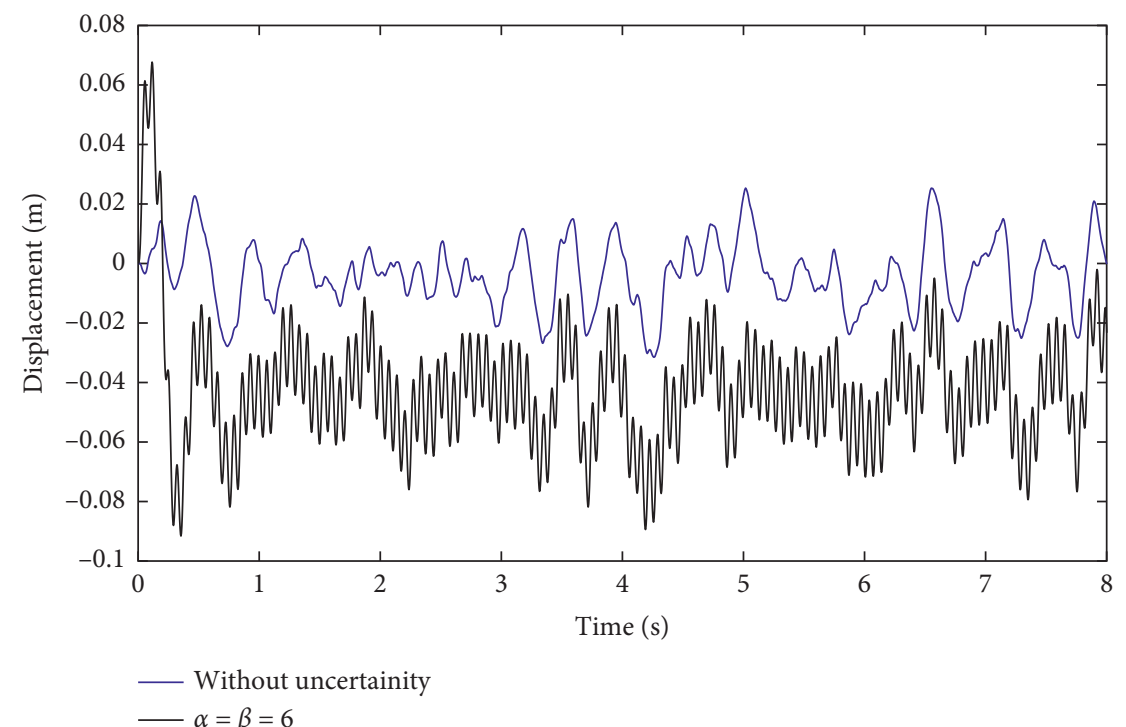

FIGURE 12: Uncontrolled system behavior in the presence of system uncertainties

maximum amount of displacement decreases with the increase of land increases.

The behavior of the controlled system for various values of $\eta$ and $\lambda=1$ is also shown in Figure 10. As evident in the figure, the amplitude of the system for $\eta=100$ is considerably higher than that at $\eta=1$ and $\eta=10$.

The maximum displacement in terms of different $\eta$ is also shown in Table 5. As can be seen from this table, the maximum amount of displacement increases with the increase of $\eta$. But as can be seen from this table, the maximum displacements for $\eta=1$ and $\eta=10$ are almost the same, but the maximum displacement for $\eta=100$ is significant.

In this part, we intend to examine controller robustness in the presence of system uncertainties. The results are obtained for $\sigma=0.05, \lambda=1$, and $\eta=10$, where $f_{1}(x, t)=$ $\alpha \times 10^{3} \sin t$ and $f_{2}(x, t)=\beta \times 10^{3} \sin (t)$. The behavior of the controlled system for different $\alpha$ and $\beta$ values is demonstrated in Figure 11. As shown by these figures, the sliding mode controller is highly capable of reducing the effects of uncertainty. 


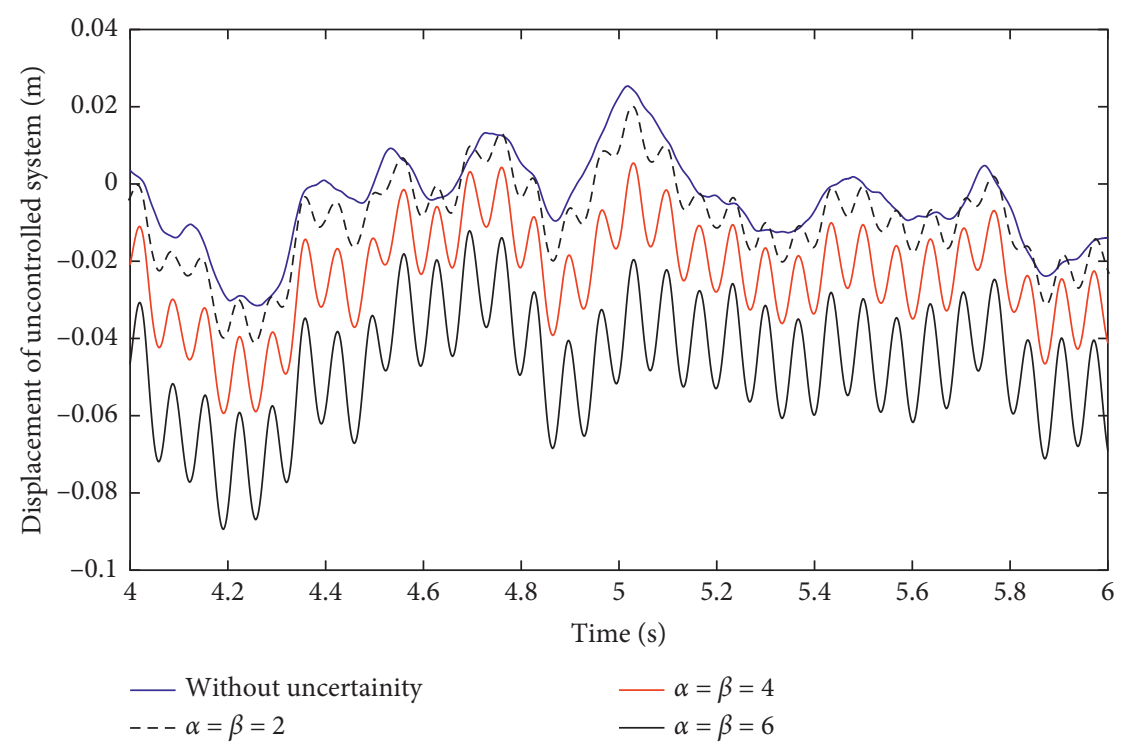

FIGURE 13: Uncontrolled system behavior in the presence of system uncertainties (detailed view).

TABle 6: Maximum displacement for various amounts of $\alpha$ and $\beta$.

\begin{tabular}{lcccc}
\hline$\alpha=\beta$ & 0 & 2 & 4 & 6 \\
\hline Maximum displacement & 0.00535 & 0.005599 & 0.006572 & 0.09158 \\
\hline
\end{tabular}

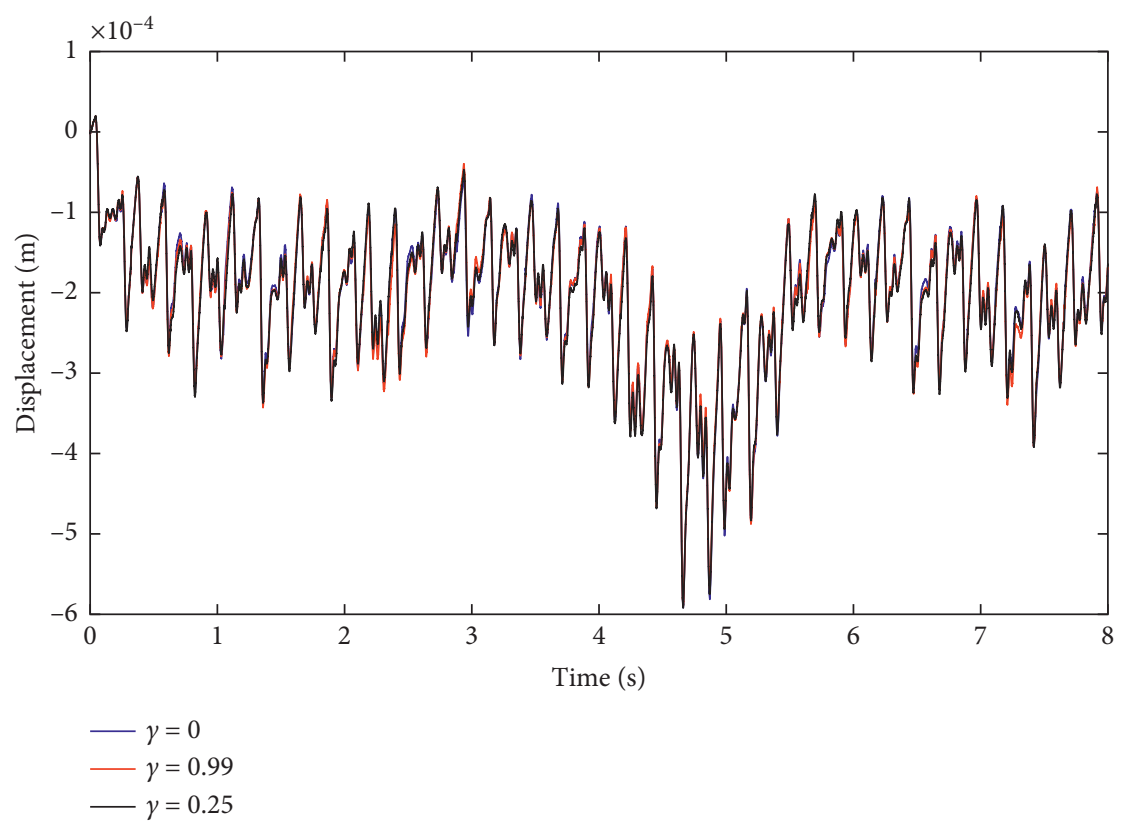

(a)

FIgURE 14: Continued. 


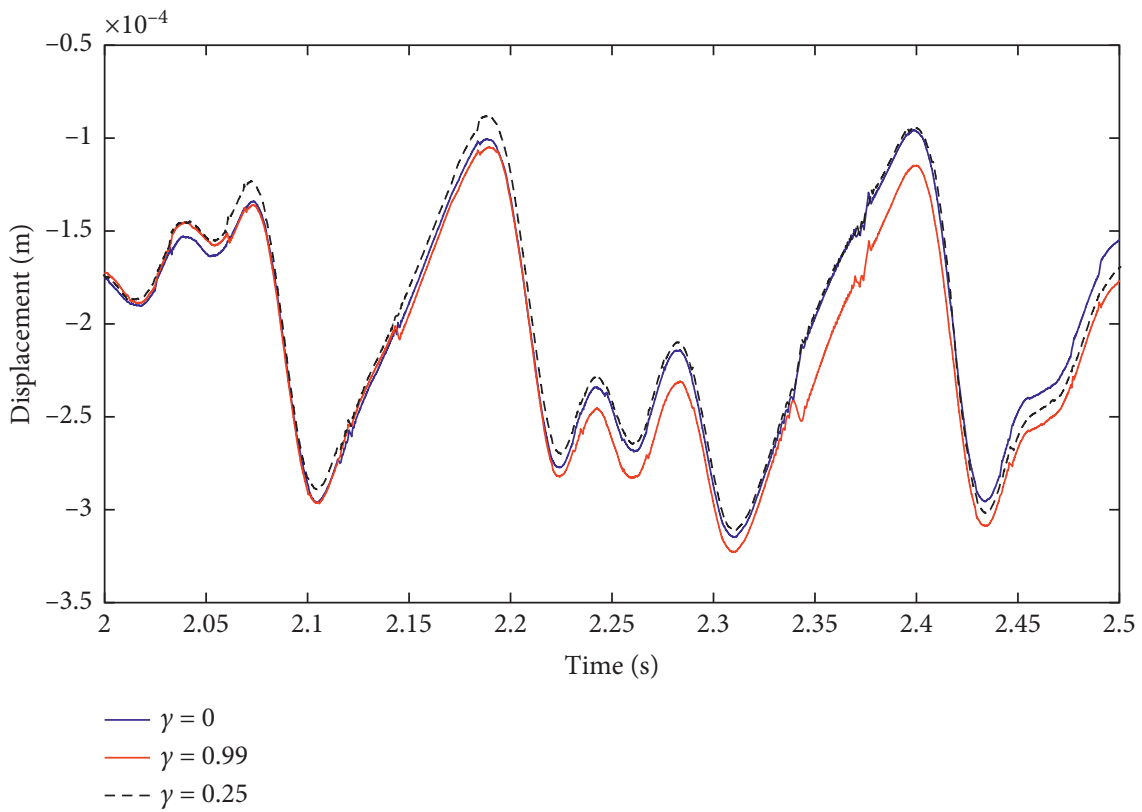

(b)

Figure 14: Controlled system behavior for different values of $\gamma$ and $\alpha=\beta=3$. (a), (b). Detailed view of Figure 13(a).

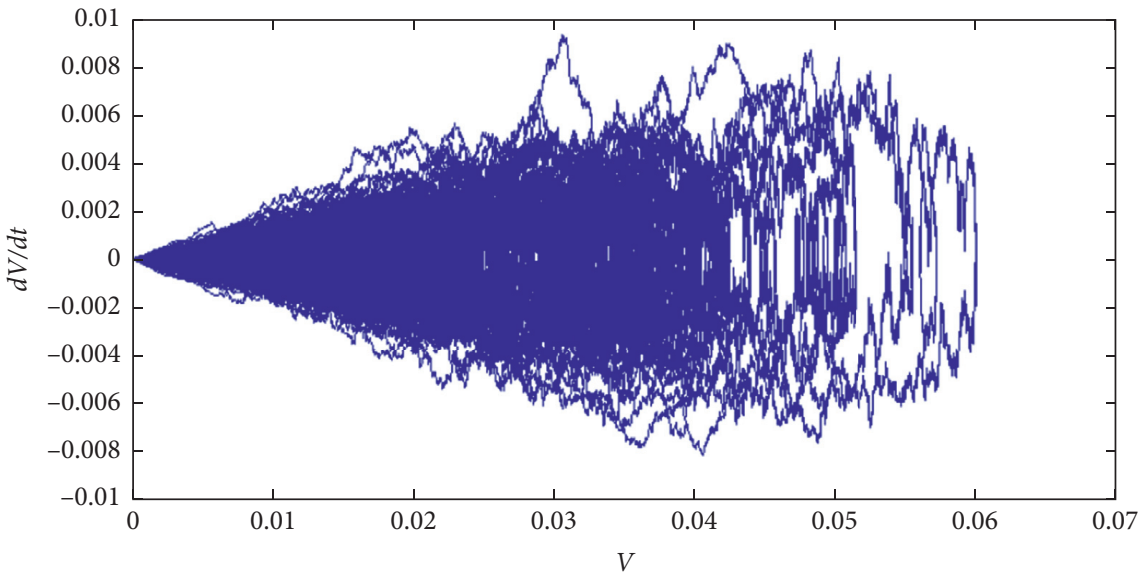

(a)

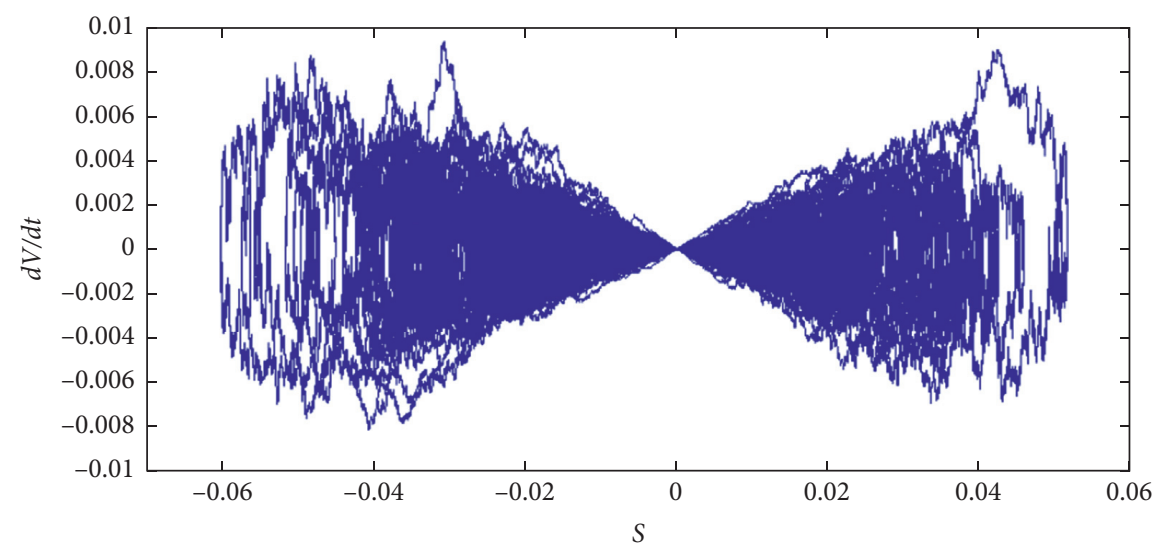

(b)

Figure 15: Variation of $\mathrm{d} V / \mathrm{d} t$ versus $V$ and $s$ for the uncontrolled system. (a). $\mathrm{d} V / \mathrm{d} t$ versus $V$ and (b). $\mathrm{d} V / \mathrm{d} t$ versus $s$. 


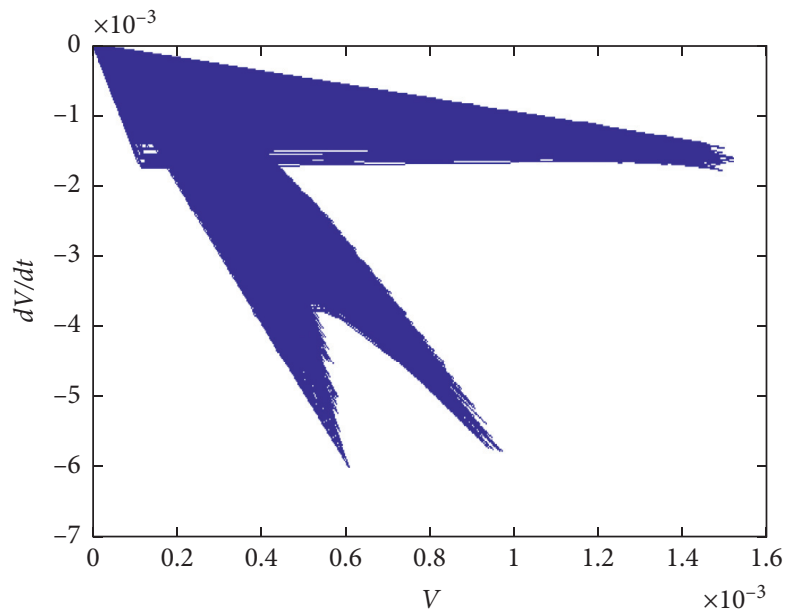

(a)

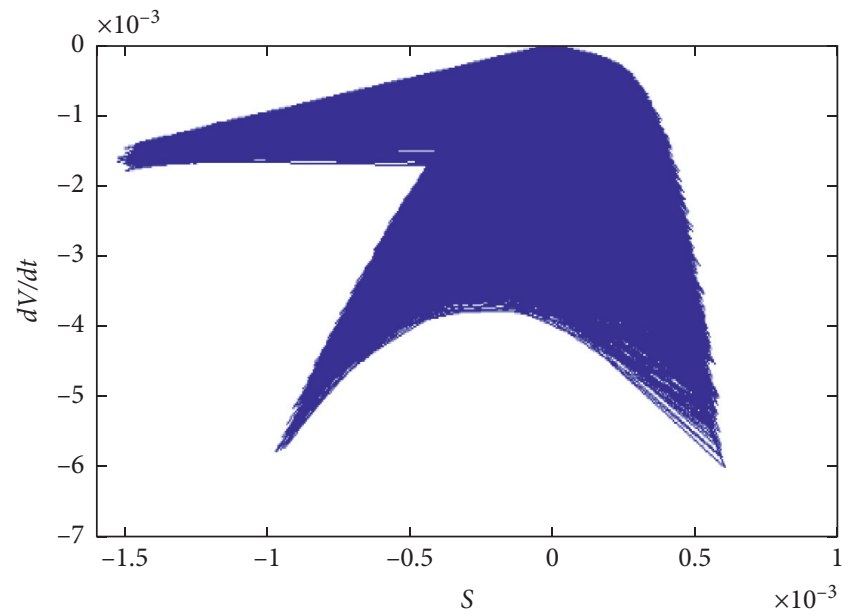

(b)

FIgure 16: $\mathrm{d} V / \mathrm{d} t$ versus $V$ and $s$ for the controlled system with $\eta=1$. (a). $\mathrm{d} V / \mathrm{d} t$ versus $V$ and (b). $\mathrm{d} V / \mathrm{d} t$ versus $s$.

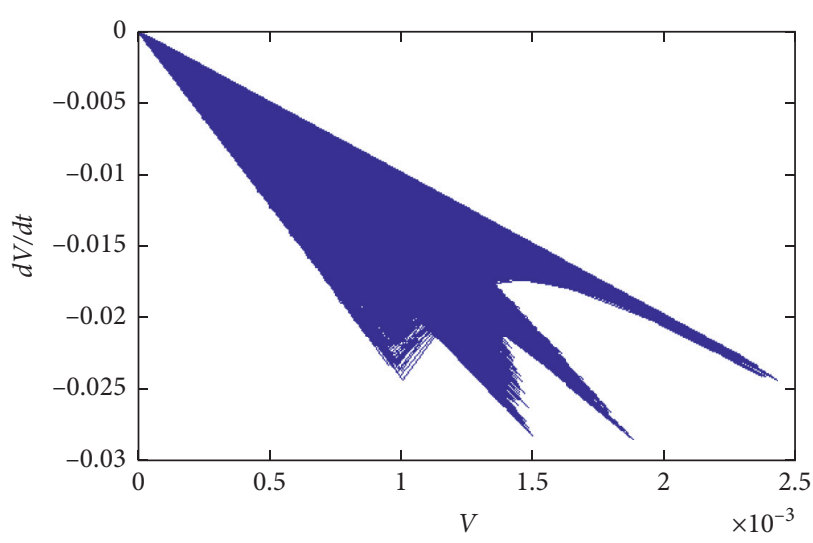

(a)

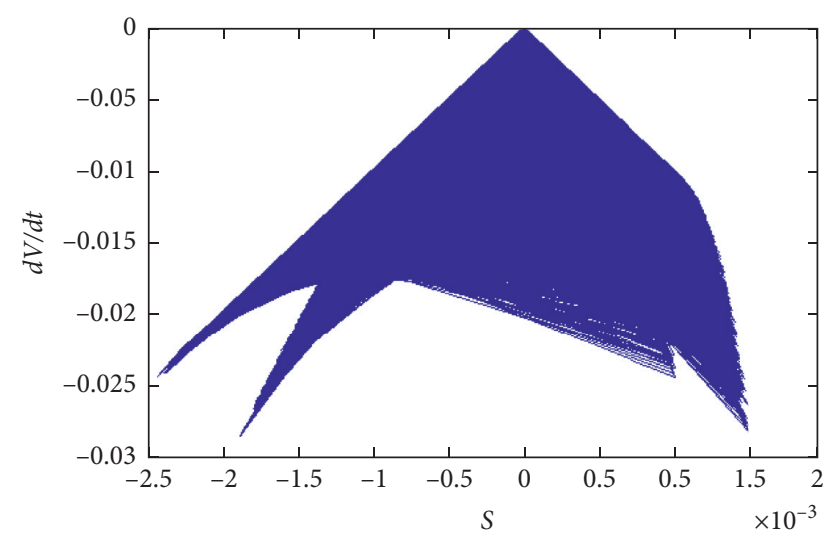

(b)

Figure 17: $\mathrm{d} V / \mathrm{d} t$ versus $V$ and $s$ for the controlled system with $\eta=10$. (a). $\mathrm{d} V / \mathrm{d} t$ versus $V$. (b). $\mathrm{d} V / \mathrm{d} t$ versus $s$.

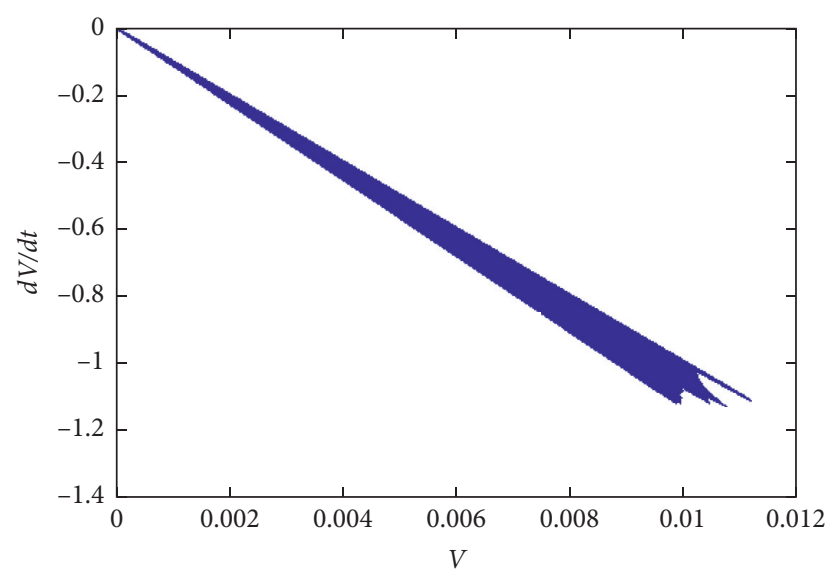

(a)

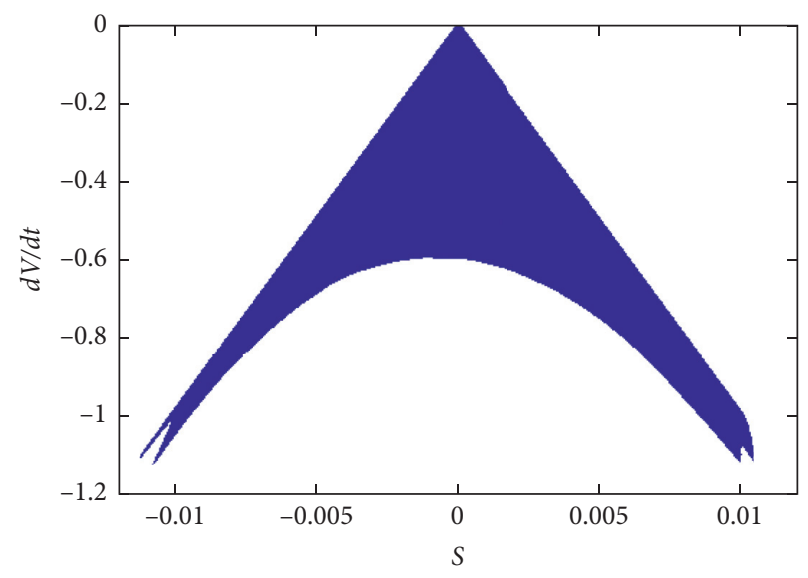

(b)

Figure 18: $\mathrm{d} V / \mathrm{d} t$ versus $V$ and $s$ for the controlled system with $\eta=100$. (a). $\mathrm{d} V / \mathrm{d} t$ versus $V$, (b). $\mathrm{d} V / \mathrm{d} t$ versus $s$. 


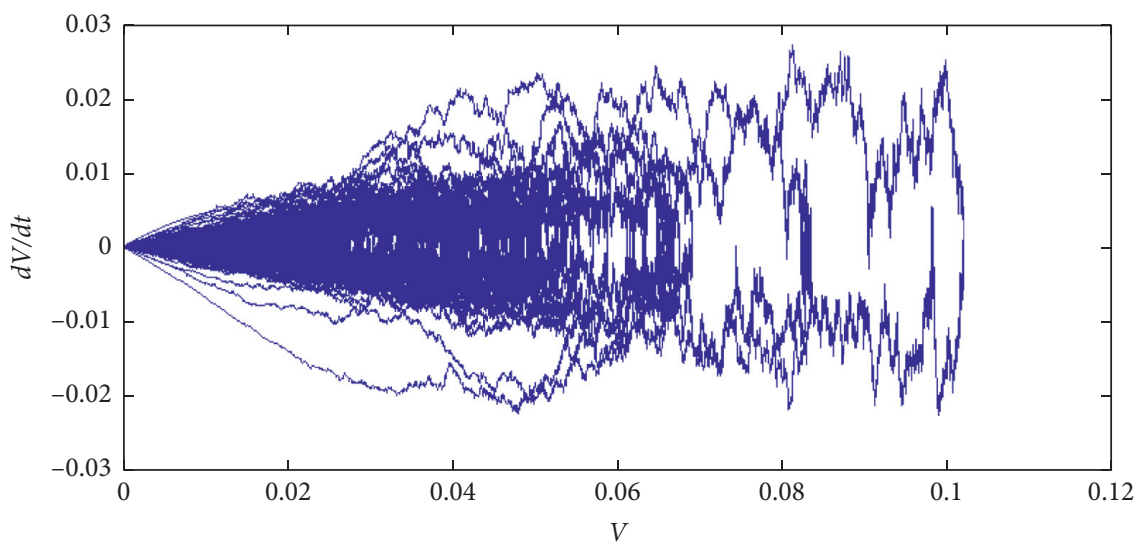

(a)

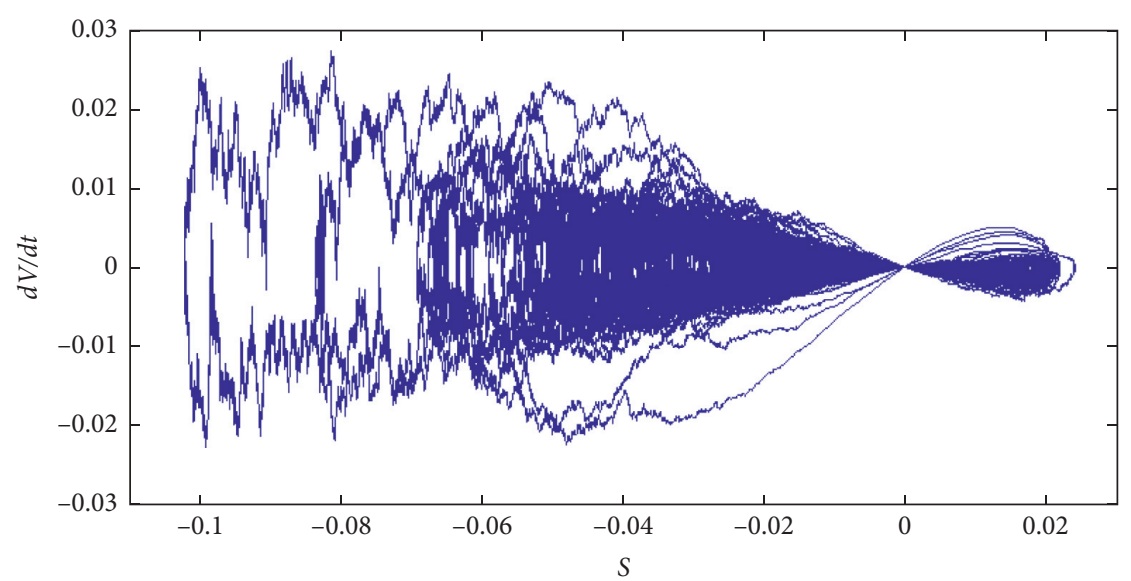

(b)

Figure 19: $\mathrm{d} V / \mathrm{d} t$ versus $V$ and $s$ for the controlled system with $\sigma=0.5$. (a) $\mathrm{d} V / \mathrm{d} t$ versus $V$ and (b) $\mathrm{d} V / \mathrm{d} t$ versus $s$.

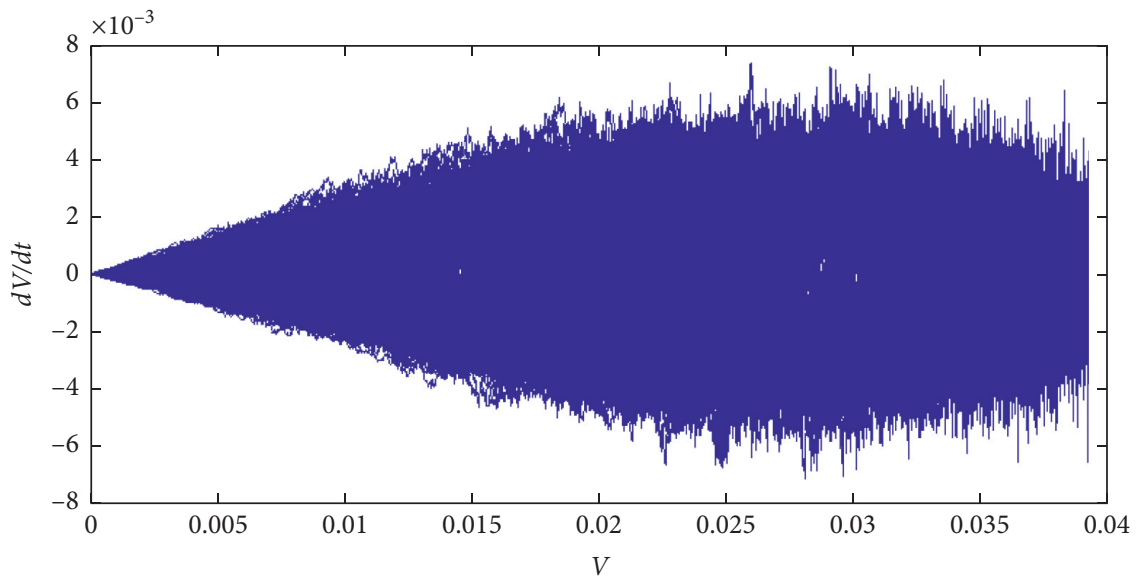

(a)

Figure 20: Continued. 


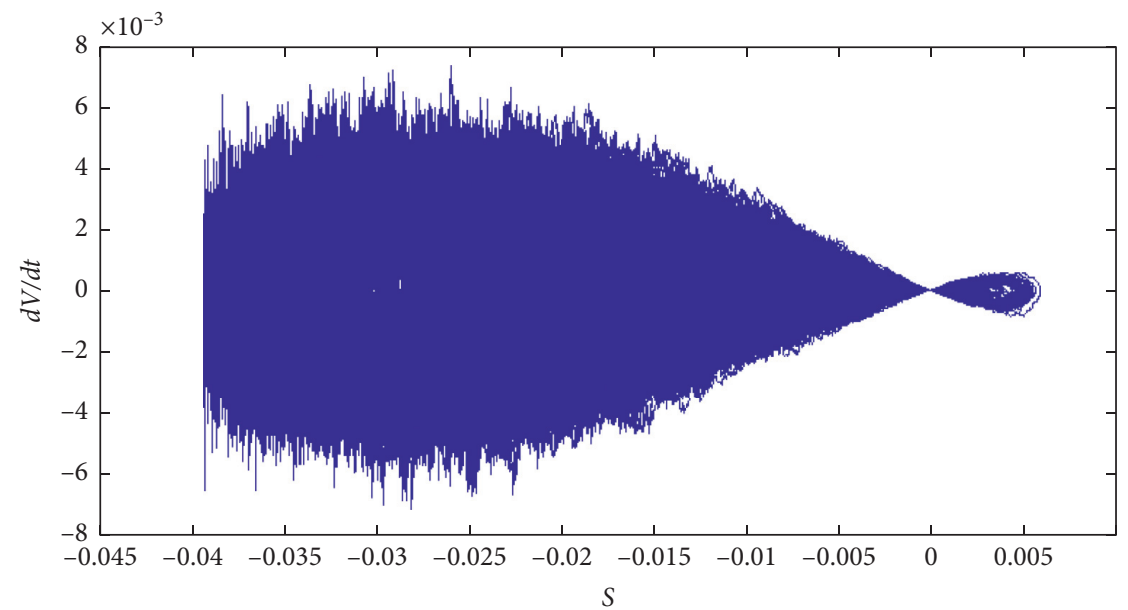

(b)

Figure 20: $\mathrm{d} V / \mathrm{d} t$ versus $V$ and $s$ for the controlled system with $\sigma=0.05$. (a) $\mathrm{d} V / \mathrm{d} t$ versus $V$ and (b) $\mathrm{d} V / \mathrm{d} t$ versus $s$.

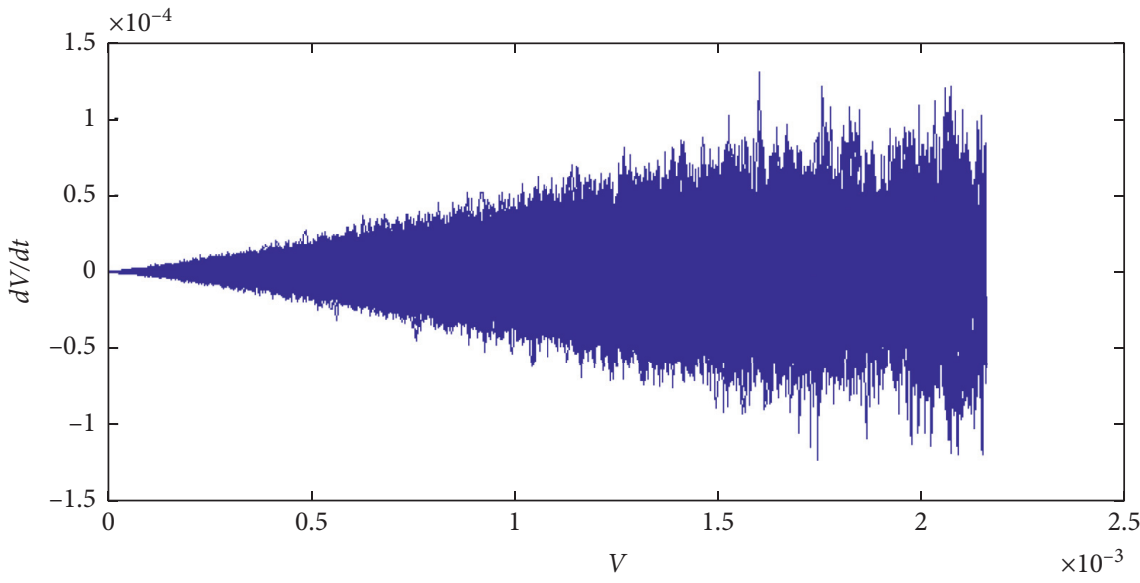

(a)

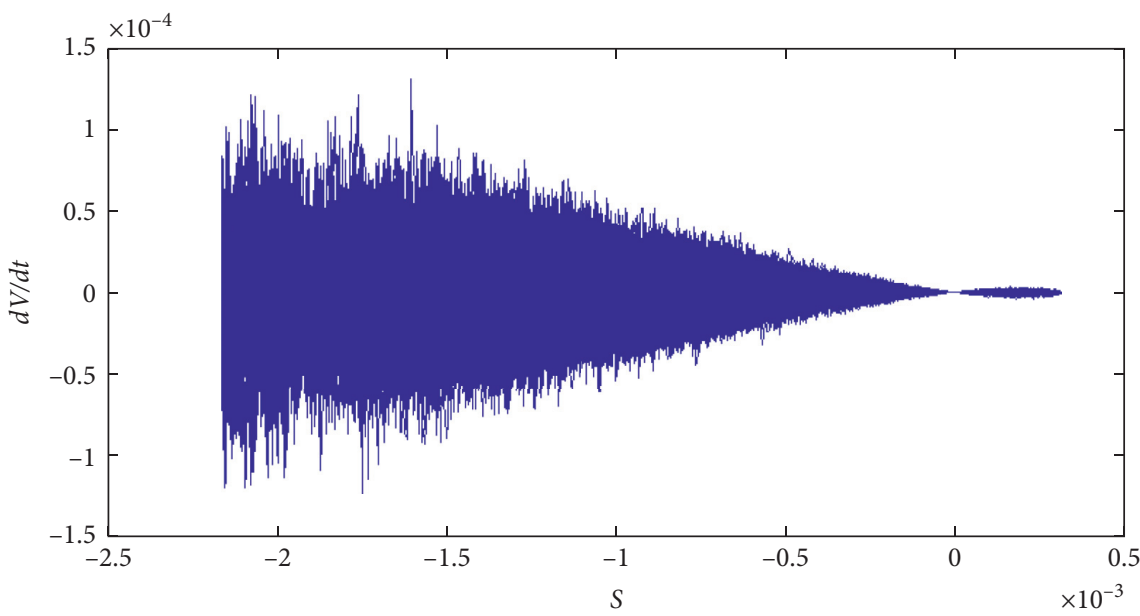

(b)

Figure 21: $\mathrm{d} V / \mathrm{d} t$ versus $V$ and $s$ for the controlled system with $\sigma=0.005$. (a) $\mathrm{d} V / \mathrm{d} t$ versus $V$ and (b) $\mathrm{d} V / \mathrm{d} t$ versus $s$. 


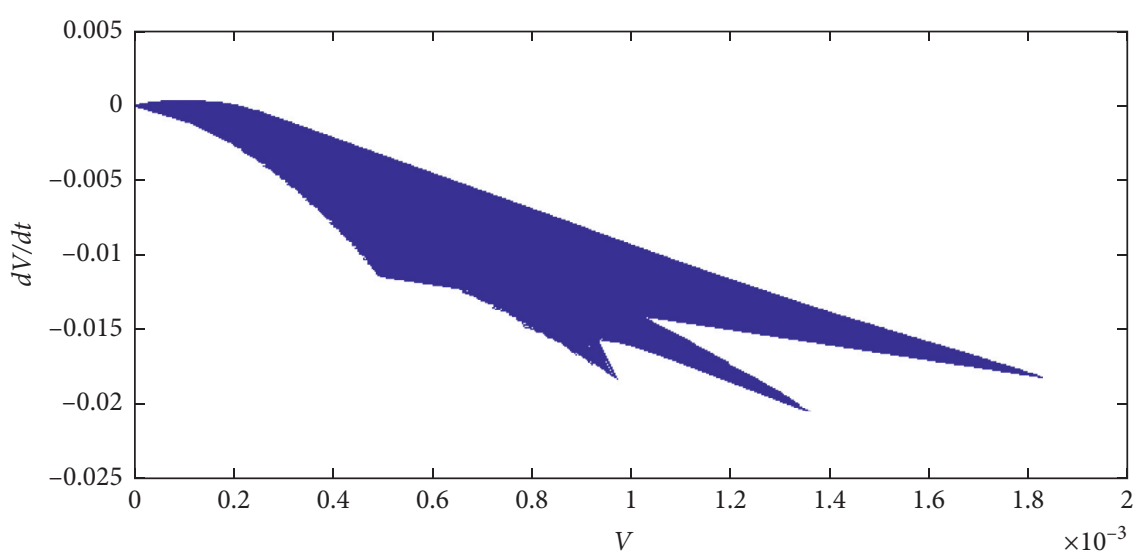

(a)

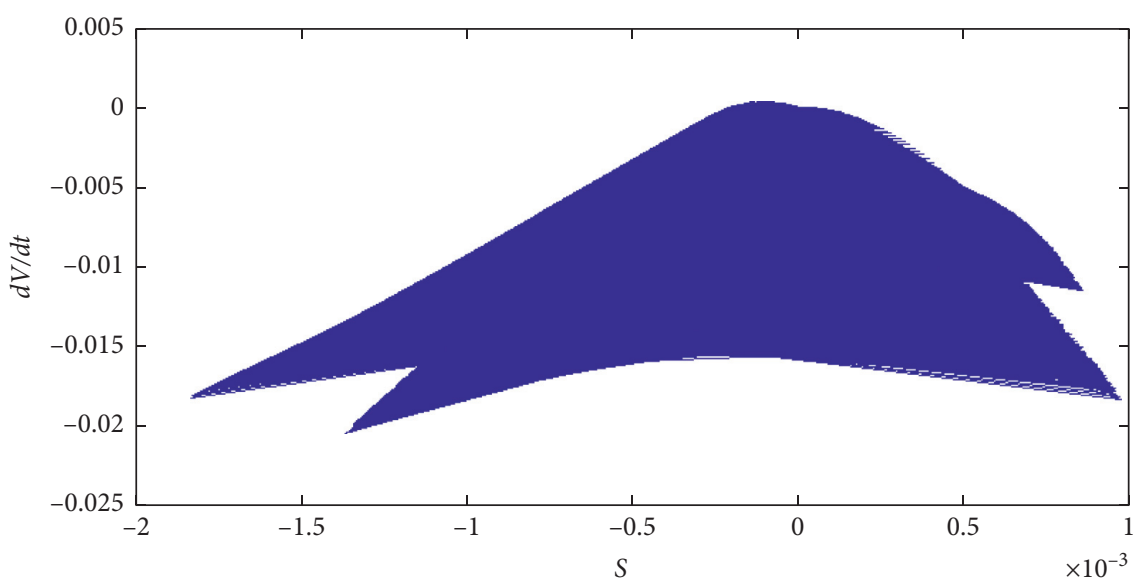

(b)

Figure 22: $\mathrm{d} V / \mathrm{d} t$ versus $V$ and $s$ for the controlled system with $\sigma=0.0005$. (a) $\mathrm{d} V / \mathrm{d} t$ versus $V$ and (b) $\mathrm{d} V / \mathrm{d} t$ versus $s$.

The behavior of the system for different values of $\alpha$ and $\beta$ in the passive case is shown in Figures 12 and 13. As shown, uncertainty significantly affects the behavior of the passive system, considerably increasing the amplitude of the system behavior.

The maximum displacement values shown in Figures 11 and 12 in terms of different $\alpha$ and $\beta$ are presented in Table 6 . As can be seen from this table, with increasing $\alpha$ and $\beta$, the maximum amount of displacement also increases. It is noteworthy that the maximum amount of displacement for the $\alpha=\beta=6$ is significant compared to other values.

In what follows, we shall discuss the controller robustness in the face of controller uncertainty and system uncertainties. For this case, it was assumed that $\alpha=\beta=3$ and $h(x, t)=1+\gamma \sin t$. The resulting system behavior is depicted in Figure 14 for different values of $\gamma$. As indicated in this figure, the controller offers high robustness in the presence of the actuator uncertainty, such that the system behavior is negligibly affected in both the absence and presence of uncertainty in the actuator. The results indicate the high robustness of the controller against actuator uncertainty.

4.2. Effect of Design Parameters and System Specifications on the Changes in the Lyapunov Function and Its Derivative.
In this section, the Lyapunov function and its dependence on parameters $\eta$ and $\sigma$ are discussed. In Figure 15(a), the diagram of $\mathrm{d} V / \mathrm{d} t$ is plotted with respect to $V$ for the passive case. As shown, the diagram $V$ always assumes positive values, but its derivative can take both positive and negative values. Note that $V=(1 / 2) s^{2}$, or in other words $s= \pm \sqrt{2 V}$. The variation of $\mathrm{d} V / \mathrm{d} t$ with respect to $s$ was used in this paper for a more accurate analysis of the results. In Figure 15(b), the diagram of $d V / d t$ is plotted with respect to $s$, which can assume both positive and negative values.

Figures 16(a), 17(a), and 18(a) depict $\mathrm{d} V / \mathrm{d} t$ in terms of $V$ for $\eta$ values of 1,10 , and 100. As shown, the Lyapunov function and its derivative always assume positive and negative values, respectively. By comparing the abovementioned figures, it follows that, as $\eta$ increases, the domain or the maximum value of the Lyapunov function and its derivative grow wider. To better illustrate this, we are going to examine this observation also on the diagram of $\mathrm{d} V / \mathrm{d} t$ with respect to $s$. Figures 16(b), 17(b), and 18(b) depict $\mathrm{d} V / \mathrm{d} t$ with respect to $s$ for $\eta$ values of 1,10 , and 100 . As shown, as $\eta$ increases, the range of variation in salso increases.

Figures $17(\mathrm{a})$ and 17 (b) show the diagrams $\mathrm{d} V / \mathrm{d} t$ with respect to $V$ and $s$. As can be seen, the range of variations in $s$ is greater for $\eta=10$ compared to $\eta=1$. The diagrams $\mathrm{d} V / \mathrm{d} t$ 


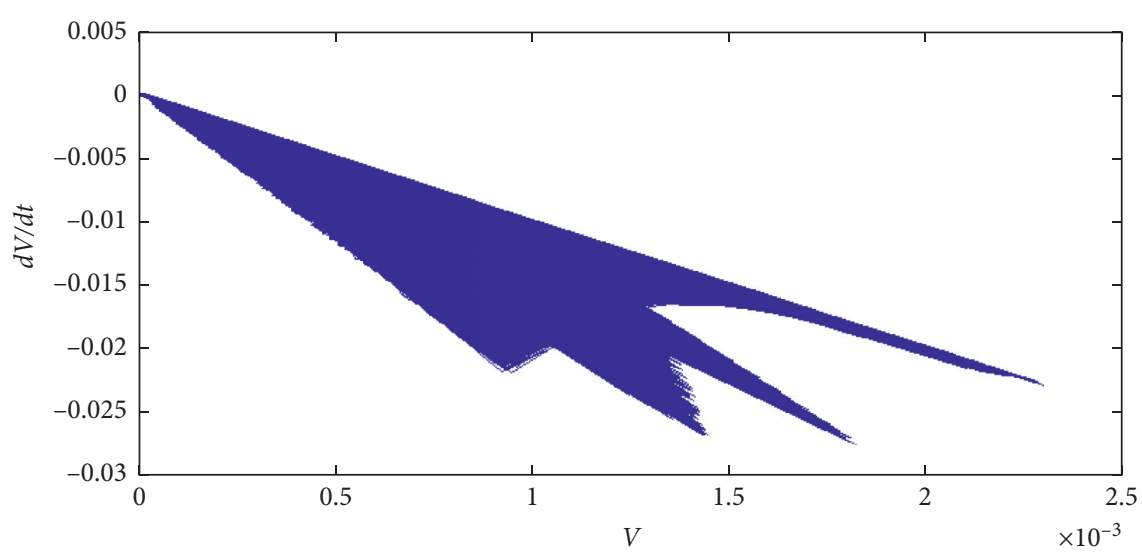

(a)

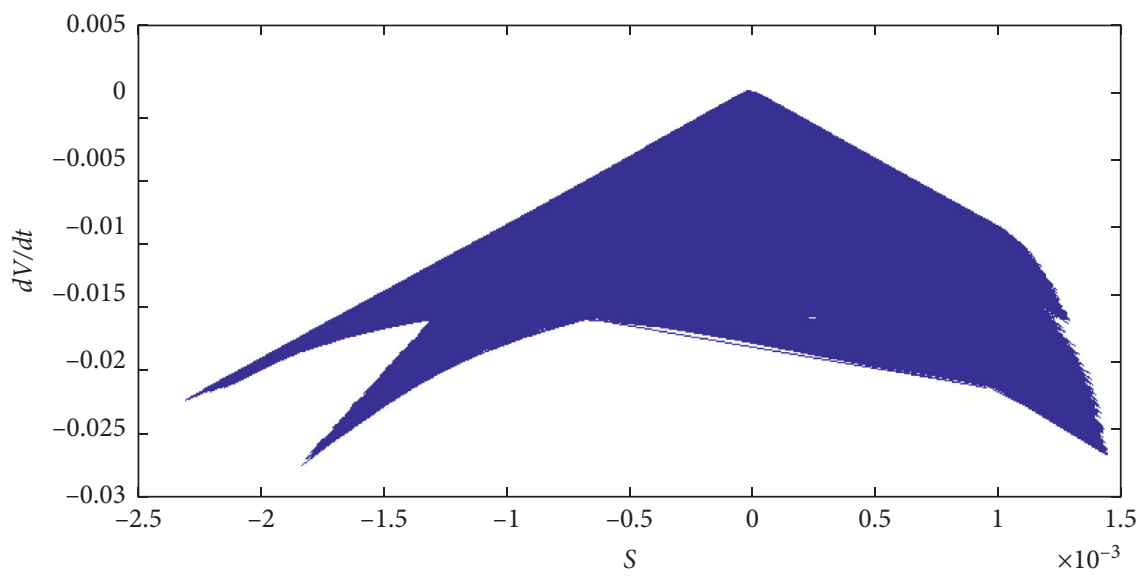

(b)

Figure 23: $\mathrm{d} V / \mathrm{d} t$ versus $V$ and $s$ for the controlled system with $\sigma=0.00005$. (a) $\mathrm{d} V / \mathrm{d} t$ versus $V$ and (b) $\mathrm{d} V / \mathrm{d} t$ versus $s$.

with respect to $V$ and $s$ for $\eta=100$ are plotted in Figures 18(a) and 18(b). As mentioned before, increasing $\eta$ increases $e_{1}$ or, in other words, according to the definition of the stability of the Lyapunov, by decreasing $\eta$, the value of $\delta(\varepsilon)$ decreases, causing the attraction set to shrink, in which case the system becomes more stable according to the definition of Lyapunov stability.

In the following section, we plan to discuss changes in $\mathrm{d} V / \mathrm{d} t$ with respect to $V$ and $s$ to eliminate chattering. As mentioned earlier, in order to eliminate the chattering phenomenon, $s /(|s|+\sigma)$ is used instead of $\operatorname{sign}(s)$, where $\sigma$ is a small number. Note that $s /(|s|+\sigma)$ is an approximation of the function sign $(s)$, the accuracy of which is acceptable if $\sigma$ is sufficiently small. However, if inappropriate values are selected for $\sigma$, the sign of $\mathrm{d} V / \mathrm{d} t$ is affected, causing it to contradict its positive definite condition. In Figures 19-23, the diagram $\mathrm{d} V / \mathrm{d} t$ with respect to $V$ and $s$ is plotted for different values of $\sigma, \lambda=1$ and $\eta=10$. A shown in Figures $19-21, \mathrm{~d} V / \mathrm{d} t$ can also take positive values, meaning that the system can become temporarily unstable from the viewpoint of the Lyapunov theory. In fact, in such conditions, the chattering phenomenon is eliminated. The diagram $\mathrm{d} V / \mathrm{d} t$ with respect to $s$ for $\sigma=0.05$ is plotted in Figure 20. As shown, the sign of the diagram $\mathrm{d} V / \mathrm{d} t$ with respect to $s$ remains unchanged in different time steps. However, as shown in Figures 22 and 23, for $\sigma=0.0005$ and $\sigma=0.00005$, the diagram $\mathrm{d} V / \mathrm{d} t$ satisfies the absolute stability condition and $\mathrm{d} V / \mathrm{d} t$ assumes negative values for all $s$ values. As previously shown, the system exhibits a chattering behavior for $\sigma=0.0005$ and $\sigma=0.00005$, since $\mathrm{d} V / \mathrm{d} t$ changes signs in each time step.

\section{Conclusion}

Given the importance of active control for car suspension systems, this paper addressed the design and dynamic analysis of a quarter-car system. To achieve more precise modeling of the suspension system, nonlinear factors in the springs and shock absorber were taken into consideration. Furthermore, uncertainty in the system and the actuator was taken into account. Road surface variations were modeled as Gaussian white noise. The sliding mode controller was employed to control the quarter-car system given that this controller is robust to uncertainty and noise and is a suitable controller for nonlinear models. The dynamic equations governing the behavior of the system were presented and the sliding mode controller was designed for the studied system. The control results for different cases of uncertainty were obtained and 
compared with the behavior of a passive system. The effect of controller parameters on the system behavior and the controller robustness to uncertainties and white noise were presented. The results show that the sliding mode controller is capable of reducing uncertainty and noise effects as well as stabilizing the system. Finally, the effects of controller parameters on the system stability from Lyapunov's view as well as on the basin attraction set were presented.

After presenting of concluding remarks, the authors are interested in presenting some future works that can be studied with the presented method of this paper.

Performing such control activity is possible in the presence of white Gaussian noise for other dynamic structures, for example, investigation of the dynamic behavior of the tall building in the presence of ground excitation or earthquake, in which the ground displacement is considered as white Gaussian noise; vibrations analysis of marine structures in the presence of storms or sea waves using the active displacement reduction system, for such research, sea waves considered as white noise; aircraft wing vibrations in the presence of wind force and reducing such vibrations by using mass or vibroimpact dampers, in which wind force can be considered as Gaussian white noise; and vibration evaluation of the dynamical system in the presence of time-delayed Markov jump.

\section{Data Availability}

The related data are presented within the manuscript.

\section{Conflicts of Interest}

The authors declare they have no conflicts of interest.

\section{Acknowledgments}

This study was funded by The Research Council of Oman under Grant no. ORG/CBS/14/008.

\section{References}

[1] E. M. Papoutsis-Kiachagias, S. Porziani, C. Groth, M. E. Biancolini, E. Costa, and K. C. Giannakoglou, "Aerodynamic optimization of car shapes using the continuous adjoint method and an RBF morpher," Computational Methods in Applied Sciences, Springer, Cham, Switzerland, pp. 173-187, 2019.

[2] S. Thomas and O. Carsten, "Adjoint optimization for vehicle external aerodynamics," International Journal of Automotive Engineering, vol. 7, no. 1, pp. 1-7, 2016.

[3] R. Boodanur, A. Panwar, S. K. Kulkarni, and A. B. Jadhav, "Air intake system optimization for passenger car engine," $S A E$ Technical Paper, 2019.

[4] Z. Xu, X. Xia, S. Lai, and Z. He, "Improvement of interior sound quality for passenger car based on optimization of sound pressure distribution in low frequency," Applied Acoustics, vol. 130, pp. 43-51, 2018.

[5] T. A. Razak, M. N. Hamid, A. M. Ghazali, S. N. Soid, and K. S. Shafee, A Stress Analysis and Design Improvement of a Car Door Hinge for Compact Cars. InEngineering Applications for New Materials and Technologies, pp. 151-161, Springer, Cham, Switzerland, 2018.
[6] J. Rios-Torres, J. Liu, and A. Khattak, "Fuel consumption for various driving styles in conventional and hybrid electric vehicles: integrating driving cycle predictions with fuel consumption optimization," International Journal of Sustainable Transportation, vol. 13, no. 2, pp. 123-137, 2019.

[7] M. Ehsani, Y. Gao, S. Longo, and K. Ebrahimi, Modern Electric, Hybrid Electric, and Fuel Cell Vehicles, CRC Press, Boca Raton, FL, USA, 2018.

[8] B. S. Kong and D. K. Park, "Design optimization of the cowl cross bar - light cowl cross bar satisfying 5 performances -," International Journal of Automotive Technology, vol. 19, no. 3, pp. 387-391, 2018.

[9] K. Suh and H. Yoon, "Design optimization of a rear independent suspension for the Korean light tactical vehicle," International Journal of Automotive Technology, vol. 19, no. 2, pp. 245-252, 2018.

[10] H. Pan and W. Sun, "Nonlinear output feedback finite-time control for vehicle active suspension systems," IEEE Transactions on Industrial Informatics, vol. 15, no. 4, pp. 2073-2082, 2018.

[11] H. Pan, X. Jing, W. Sun, and H. Gao, "A bioinspired dynamics-based adaptive tracking control for nonlinear suspension systems," IEEE Transactions on Control Systems Technology, vol. 26, no. 3, pp. 903-914, 2017.

[12] H. Pan, H. Li, W. Sun, and Z. Wang, "Adaptive fault-tolerant compensation control and its application to nonlinear suspension systems," IEEE Transactions on Systems, Man, and Cybernetics: Systems, vol. 50, no. 5, pp. 1766-1776, 2018.

[13] M. Nagarkar, Y. Bhalerao, G. V. Patil, and R. Z. Patil, "Multiobjective optimization of nonlinear quarter car suspension system - PID and LQR control," Procedia Manufacturing, vol. 20, pp. 420-427, 2018.

[14] Y. Qin, C. Xiang, Z. Wang, and M. Dong, "Road excitation classification for semi-active suspension system based on system response," Journal of Vibration and Control, vol. 24, no. 13, pp. 2732-2748, 2018.

[15] Y. Xia, M. Fu, C. Li, F. Pu, and Y. Xu, "Active disturbance rejection control for active suspension system of tracked vehicles with gun," IEEE Transactions on Industrial Electronics, vol. 65, no. 5, pp. 4051-4060, 2017.

[16] S. M. H. Rizvi, M. Abid, A. Q. Khan, S. G. Satti, and J. Latif, "Hocontrol of 8 degrees of freedom vehicle active suspension system," Journal of King Saud University - Engineering Sciences, vol. 30, no. 2, pp. 161-169, 2018.

[17] H. P. Wang, G. I. Y. Mustafa, and Y. Tian, "Model-free fractional-order sliding mode control for an active vehicle suspension system," Advances in Engineering Software, vol. 115, pp. 452-461, 2018.

[18] A. Mardani, "Energy harvesting, handling and ride comfort trade-off between passive and active suspension systems of half vehicle model using PID controller for off-road vehicles," Journal of Advances in Vehicle Engineering, vol. 3, pp. 150-160, 2017.

[19] H. Khodadadi and H. Ghadiri, "Self-tuning PID controller design using fuzzy logic for half car active suspension system," International Journal of Dynamics and Control, vol. 6, no. 1, pp. 224-232, 2018.

[20] Y. Jiang, S. Yin, J. Dong, and O. Kaynak, “A review on soft sensors for monitoring, control and optimization of industrial processes," IEEE Sensors Journal, 2020.

[21] Y. Jiang and S. Yin, "Recursive total principle component regression based fault detection and its application to vehicular cyber-physical systems," IEEE Transactions on Industrial Informatics, vol. 14, no. 4, pp. 1415-1423, 2017. 
[22] Y. Wang, Y. Xia, H. Li, and P. Zhou, "A new integral sliding mode design method for nonlinear stochastic systems," Automatica, vol. 90, pp. 304-309, 2018.

[23] Y. Wang, X. Xie, M. Chadli, S. Xie, and Y. Peng, "Sliding mode control of fuzzy singularly perturbed descriptor systems," IEEE Transactions on Fuzzy Systems, p. 1, 2020.

[24] H. Mobki, A. M. Sabegh, A. Azizi, and H. M. Ouakad, "On the implementation of adaptive sliding mode robust controller in the stabilization of electrically actuated micro-tunable capacitor," Microsystem Technologies, vol. 26, no. 12, pp. 3903-3916, 2020.

[25] M. P. Aghababa, "A fractional sliding mode for finite-time control scheme with application to stabilization of electrostatic and electromechanical transducers," Applied Mathematical Modelling, vol. 39, no. 20, pp. 6103-6113, 2015.

[26] M. P. Aghababa, "Synchronization and stabilization of fractional second-order nonlinear complex systems," Nonlinear Dynamics, vol. 80, no. 4, pp. 1731-1744, 2015.

[27] S. Mirzajani, M. P. Aghababa, and A. Heydari, "Adaptive T-S fuzzy control design for fractional-order systems with parametric uncertainty and input constraint," Fuzzy Sets and Systems, vol. 365, pp. 22-39, 2019.

[28] M. P. Aghababa and S. Moradi, "Robust adaptive dynamic surface back-stepping tracking control of high-order strictfeedback nonlinear systems via disturbance observer approach," International Journal of Control, vol. 4, pp. 1-17, 2020.

[29] A. M. Shotorbani, A. Ajami, S. G. Zadeh, M. P. Aghababa, and B. Mahboubi, "Robust terminal sliding mode power flow controller using unified power flow controller with adaptive observer and local measurement," IET Generation, Transmission \& Distribution, vol. 8, no. 10, pp. 1712-1723, 2014.

[30] H. Zhang, X. Liu, J. Wang, and H. R. Karimi, "Robust Ho sliding mode control with pole placement for a fluid power electrohydraulic actuator (EHA) system," The International Journal of Advanced Manufacturing Technology, vol. 73, no. 5-8, pp. 1095-1104, 2014.

[31] A. Azizi, "Computer-based analysis of the stochastic stability of mechanical structures driven by white and colored noise," Sustainability, vol. 10, no. 10, p. 3419, 2018.

[32] A. Azizi, "A case study on computer-based analysis of the stochastic stability of mechanical structures driven by white and colored noise: utilizing artificial intelligence techniques to design an effective active suspension system," Complexity, vol. 25, p. $8,2020$.

[33] A. Azizi and P. Ghafoorpoor Yazdi, "Modeling and control of the effect of the noise on the mechanical structures," in Computer-Based Analysis of the Stochastic Stability of Mechanical Structures Driven by White and Colored Noise, pp. 75-93, Springer, Singapore, 2019.

[34] A. Azizi and P. Ghafoorpoor Yazdi, "Noise control techniques," in Computer-Based Analysis of the Stochastic Stability of Mechanical Structures Driven by White and Colored Noise, pp. 61-73, Springer, Singapore, 2019.

[35] M. P. Aghababa, "Comments on "Fuzzy fractional order sliding mode controller for nonlinear systems," Communications in Nonlinear Science and Numerical Simulation, vol. 17, no. 3, pp. 1489-1492, 2012.

[36] H. Yang, Y. Jiang, and S. Yin, "Fault-tolerant control of timedelay Markov jump systems with \$Ithat $\{0\}$ stochastic process and output disturbance based on sliding mode observer," IEEE Transactions on Industrial Informatics, vol. 14, no. 12, pp. 5299-5307, 2018.
[37] M. F. Ismail, K. Peng, N. Hamzah, Y. M. Sam, M. K. Aripin, and M. H. Hasan, "A linear model of quarter car active suspension system using composite nonlinear feedback control," in Proceedings of the 2012 IEEE Student Conference on Research and Development (SCOReD), pp. 98-103, Pulau Pinang, Malaysia, 2012.

[38] N. Ishak, R. S. Othman, A. Ahmad, Y. M. Sam, and A. A. Basari, "An observer design of nonlinear quarter car model for active suspension system by using backstepping controller," in Proceedings of the 2009 5th International Colloquium on Signal Processing \& Its Applications, pp. 160165, Kuala Lumpur, Malaysia, 2009.

[39] H. Mobki, M. Jalilirad, M. V. Moradi, and A. Azizi, "Multi input versus single input sliding mode for closed-loop control of capacitive micro structures," SN Applied Sciences, vol. 1, no. 7, pp. 1-13, 2019.

[40] D. J. Higham., "An algorithmic introduction to numerical simulation of stochastic differential equations," SIAM Review, vol. 43, no. 3, pp. 525-546, 2001.

[41] A. Ajami, A. M. Shotorbani, and M. P. Aagababa, “Application of the direct Lyapunov method for robust finite-time power flow control with a unified power flow controller," IET Generation, Transmission \& Distribution, vol. 6, no. 9, pp. 822-830, 2012.

[42] M. P. Aghababa, "Lyapunov control method for mismatched uncertainty and gain variation compensation in switched systems," IEEE Transactions on Systems, Man, and Cybernetics: Systems, pp. 1-11, 2020.

[43] M. P. Aghababa and H. Feizi, "Nonsingular terminal sliding mode approach applied to synchronize chaotic systems with unknown parameters and nonlinear inputs," Chinese Physics $B$, vol. 21, no. 6, Article ID 060506, 2012.

[44] A. Medio and M. Lines, Nonlinear Dynamics: A Primer, Cambridge University Press, Cambridge, UK, 2001.

[45] J. J. Slotine and W. Li, Applied Nonlinear Control, PrenticeHall, Englewood Cliffs, NJ, USA, 1991.

[46] H. Salarieh and A. Alasty, "Control of stochastic chaos using sliding mode method," Journal of Computational and Applied Mathematics, vol. 225, no. 1, pp. 135-145, 2009.

[47] A. S. Poznyak, "Stochastic sliding mode control and state estimation," in Advances in Variable Structure Systems and Sliding Mode Control - Theory and Applications, pp. 57-100, Springer, Cham, Switzerland, 2018.

[48] A. Poznyak, "Stochastic super-twist sliding mode controller," IEEE Transactions on Automatic Control, vol. 63, no. 5, pp. 1538-1544, 2018.

[49] E. R. Da Silva, E. Assunção, M. Teixeira, and L. F. Buzachero, "Less conservative control design for linear systems with polytopic uncertainties via state-derivative feedback," Mathematical Problems in Engineering, vol. 2012, Article ID 315049, 21 pages, 2012.

[50] S. Boyd, L. El Ghaoui, E. Feron, and V. Balakrishnan, "Linear matrix inequalities in system and control theory," Society For Industrial And Applied Mathematics, 1994.

[51] P. Gahinet, A. Nemirovskii, A. J. Laub, and M. Chilali, "The LMI control toolbox," in Proceedings of $199433 \mathrm{rd}$ IEEE Conference on Decision and Control, vol. 3, pp. 2038-2041, Lake Buena Vista, FL, USA, 1994. 